\title{
Linear Neural Circuitry Model for Visual Receptive Fields
}

\author{
Sasan Mahmoodi \\ School of Electronic and Computer Science, University of Southampton, UK \\ sm3@ecs.soton.ac.uk, Tel: +44 (0)238059 3284
}

\begin{abstract}
The current state of art in the literature indicates that linear visual receptive fields are Gaussian or formed based on Gaussian kernels in biological visual systems. In this paper, by employing hypotheses based on the anatomy and physiology of vertebrate biological vision, we propose a neural circuitry possessing Gaussian-related visual receptive fields. Here we therefore present a plausible circuitry system matching the characteristic properties of an ideal visual front end of biological visual systems and then present a condition under which this circuit demonstrates a linear behavior to model the linear receptive fields observed in the biological experimental data. The objective of this study is to understand the hardware circuitry from which various visual receptive fields in biological visual system can be deduced. In our model, a nonlinear neural network communicating with spikes is considered. The condition under which this neural network behaves linearly is discussed. The equivalent linear circuit proposed here employs some anatomical and physiological properties of the early biological visual pathway to derive the visual receptive field profiles for linear cells such as neurons with isotropic separable, non-isotropic separable and non-separable (velocity-adapted) Gaussian receptive fields in the LGN and striate cortex. In the model presented here, the theory of transmission lines for linear distributed electrical circuits are employed for two dimensional transmission grids to model cell connectivities in a neural layer. The model presented here leads to a formulation similar to the Gaussian scale space theory for the transmission of visual signals through various layers of neurons. Our model therefore presents a new insight on how the convolution process with Gaussian kernels can be
\end{abstract}


implemented in vertebrate visual systems. The comparison of the numerical simulations of our model presented in this paper with the data analysis of receptive field profiles recorded in the biological literature demonstrates a complete agreement between our theoretical model and experimental data. Our model is also in good agreement with the numerical results of the Gaussian scale space theory for the visual receptive fields.

Keywords: Gaussian Filtering; Neural Layers, Visual Receptive Fields, Linear Cells, Nonlinear Neural Networks

\section{Introduction}

The concept of receptive fields is initially introduced to explain reflexes produced by a stimulus in the somatosensory area of a body surface (Sherrington 1906). This notion is then extended in (Hartline 1938) to light stimuli to specify visual receptive fields, as a visual field region in which if visual stimuli are presented, the cell corresponding to the receptive field, responds. "ON" and "OFF" sub-regions within visual receptive fields are then discovered by Kuffler (1953). The orientation tuning of neurons in the primary visual cortex is then uncovered by Hubel and Wiesel who characterize the neurons' responses in the primary visual cortex (V1) by considering the cells' responses to the polarity of visual stimuli (Hubel and Wiesel 2005). Later DeAngelis et al. (DeAngelis et al. 1995; DeAngelis and Anzai 2004) employ receptive field mapping techniques based on white noise stimuli to characterize the neurons' responses in the joint space-time domain. The spatio-chromatic and spatio-chromatemporal responses are then described by Conway and Livingstone (2006). A spatio-temporal energy model for motion perception is also developed in (Adelson and Bergen 1985) by exploiting oriented filters in the space-time domain. Spatio-temporal receptive fields are also modelled in (Young et al. 2001) by using Gaussian derivatives over a joint space-time domain. In fact, biological experiments quantitatively indicate that the linear visual receptive fields are well-modelled as mainly Gabor kernels, differences of Gaussians and Gaussian 
derivatives. The receptive fields as Gaussian related kernels in biological vision are considered as tools for canonical neural computations of the brain as suggested by physiological and behavioural evidence (Carandini and Heeger 2012).

On the other hand, scale space theory has been developed to provide a general framework for early visual operations in any universal visual front end (see e.g. Weickert et al. 1999; Lindeberg 2011). Gaussian kernel and its family are derived by postulating a set of mathematical properties (axioms) which an early visual system is expected to possess (see e.g. Weickert et al. 1997; Florack 1997; ter Haar Romeny 2003). In (Florack et al. 1992), it is demonstrated that the class of admissible scale-space kernels can be confined by including semi-group, scale invariance and rotational symmetry properties. The separability of a kernel in Cartesian coordinates then leads to Gaussian scale space. Gaussian scale space is also introduced for temporal data in (Koenderink 1988) and further investigated in (Florack 1997; ter Haar Romeny et al. 2001). A time-recursive space time separable spatio-temporal scale space model is then developed in (Lindeberg and Fagerstrom 1996). A Poisson scale space formulation which does not possess the property of the non-enhancement of local exterma, is also investigated in (Duits et al. 2004). Gaussian scale space framework is also employed in (Lindeberg 2011; Lindeberg 2013) to present 1) a continuous time-causal scale-space model, 2) a time recursive update mechanism, 3) a parameterization of the spatio-temporal filters with respect to image velocity and image deformation and 4) convincing results from the scale-space models determined by a set of structural constraints for an idealized vision system. Early biological visual systems possessing the mathematical properties suggested in Gaussian scale space theory, are also well known to be associated with the Gaussian related kernels. It is noted that Gaussian scale space theory has been developed in a mathematical setting for any general early visual system which possesses a set of mathematical properties. The hypothesis is that since the early biological visual system enjoys these mathematical 
properties, it should also possess Gaussian related kernels. The fact that the numerical results of the kernels derived in the Gaussian scale space framework are in agreement with linear receptive fields in vertebrate early visual systems (Lindeberg 2011; Lindeberg 2013), confirms this hypothesis. However, no anatomical, and nor physiological assumptions for early biological visual systems are considered for the derivations of the Gaussian related kernels in a Gaussian scale-space setting.

In this paper, a model based on distributed electrical circuits is proposed to formulate electrical connectivity of neurons in retina and other following neural layers as two dimensional distributed linear circuits in the visual pathway. Neurons in a given layer send trains of spikes to neurons in the next layer through their axons, if their membrane potential is greater than a certain threshold. In such a scenario, according to classical rectification model (Granit et al. 1963; Carandini and Fester 2000), low pass signals (membrane potentials) in a neuron is linearly associated with the firing rate of neurons, if the potential is above a certain threshold. It is important to notice that the input of any single neuron in a given layer is connected both directly to the output of the corresponding neuron in the previous layer and indirectly to the outputs of all other neurons in the previous layer through the conductive sheet. The circuit proposed here is nonlinear in nature. We therefore present a condition under which this circuit behaves linearly. A linear electrical circuit equivalent to this nonlinear neural circuitry is proposed in this paper to facilitate the derivations of the visual receptive fields. In such an equivalent linear circuit, a neuron in a given layer is directly connected to its counterpart in the next layer so that the membrane potential of a neuron is directly and linearly affected by the membrane potential of the corresponding neuron in the previous layer. Our contributions in this paper are as follows:

1) Here we propose an electrical circuit, based on the anatomy and physiological properties of early visual systems to model the neural connectivity in this visual path. 
2) In contrast with Gaussian scale space frameworks, linearity is not one of our assumptions. Our model is hence nonlinear in nature. We therefore show here that this nonlinear system behaves like a linear one under some certain conditions.

3) Here we demonstrate that the within-dendritic-network processes produce the Gaussian aspect of the visual receptive fields. This is in contrast with the previous work (see e.g (von Seelen et al. 1987; Dinse and von Seelen 1981; Krone et al. 1986)) in the literature where the Gaussian distributed synaptic connections are considered the reason for the Gaussian aspect of the visual receptive fields.

4) In our numerical results, it is demonstrated that non-isotropic-elongated receptive fields are better matched with the receptive fields of a group of isotopic Gaussian neurons rather than with a single neuron possessing a non-isotropic-elongated Gaussian receptive field.

5) In our model, we show that the effects seen in the causal temporal smoothing of spatiotemporal (separable and non-separable) receptive fields are produced by the neuron's axons behaving like transmission lines. It is demonstrated here that these cascaded neural axons, also proposed in a simultaneous and independent research in (Lindeberg 2015) as a serially coupled first order integrators, produce numerical results very similar to the biological recordings.

6) Last but not least, as mentioned before, we have here started with a small set of hypotheses based on the anatomy and physiology of the early biological visual system. Gaussian related kernels are then derived to describe the behaviour of the visual receptive fields of cells in this visual system. This is in contrast with the scale-space framework where a set of mathematical axioms (requirements) for a visual system is the base for scale-space theory. It is important to notice that in our derivation, no axiom from Gaussian scale space framework is used. From the standpoint of the philosophy of science, this is important and interesting. Gaussian scale-space formulation has been developed in a 
mathematical setting (mathematical world) starting with some general (mathematical) properties for a universal visual system. Yet, this formulation connects nicely with a biological visual system in our physical world (see Penrose 2004, section 1.4 for discussions on mathematical and physical worlds) through the comparison between the numerical results of the theory and biological experiments. On the other hand, only anatomical and physiological assumptions (in the physical world) are made in this paper in our model. Our numerical results are also in good agreement with biological experiments. Finally we also show here that our model which is based on physicalbiological assumptions is in good agreement with the completely mathematically derived Gaussian scale-space theory.

The rest of this paper is structured as follows. In section 2, the model proposed here is described and its mathematical formulation and applications in vertebrate early visual processing are discussed. In section 3 , the properties of our model as an ideal biological visual system are investigated and the numerical results of our model are presented. In this section, we also theoretically demonstrate that our model meets the structural requirements of scale space theory. Finally conclusions are drawn in section 4.

\section{Model for Early Visual Pathway}

\subsection{Model Hypotheses}

Our model for early visual pathway presented in this paper is based on four main hypotheses based on the anatomy and physiological properties of biological visual systems. We describe these hypotheses in this section and explain our rationales behind them.

1) Our first assumption here is that early visual processing is performed by a number of neural layers connected serially together. Our hypothesis that the visual information 
passes through a series of neural layers before the visual signals reach the visual cortex is supported by the fact that retina itself consists of four distinctive neural layers (four sets of synapses) as follows (Caceci 1998): Ganglion cell layer, Inner nuclear layer consisting of horizontal, bipolar and amarine cells, photoreceptors layer containing rod and cone cells and retinal pigment epithelium consisting of cuboidal cells. These four retinal layers are then followed by a few neural layers in LGN. Again in the striate cortex, our assumption is that the visual signal is transmitted through a number of serially cascaded layers of neurons.

2) Our second assumption here is that we model any layer of neurons in early biological vision starting from retina's photoreceptors as a two dimensional distributed conductive grid conducting dendritic potentials in two dimensional conductors consisting of resistors, and capacitors. In fact, this conductive grid consists of millions of dendritic spines inter-connected together through dendrite membranes (Baer and Rinzel 1991). A layer of neurons in early visual pathway therefore is connected to the previous layer through dendritic spine heads which are also interconnected through the dendrites. We therefore model these connectivities within a layer of neurons as a two dimensional distributed conductive grid. The membrane dendritic potentials are transmitted from neurons in one layer to those in the next layer in the form of the transmission of spike pulses. Here, we generalize the theory of transmission lines (Skilling 1979) to continuous two dimensional grid conductors. The dendritic spine heads are characterized with high input resistance (Baer and Rinzel 1991). In our investigation here, we further assume that these neural layers modelled with electrically conductive layers in the retina have similar and analogous electrical structures but they may possess different electrical parameters.

3) Neural layers are rotationally symmetric. This is to say that by rotating a layer, the 
electrical properties of the layer remain unchanged along a certain orientation.

4) Our final assumption is that the distance between neighboring neurons is negligible in comparison with the dimensions of the visual field in the retina or any neural layer.

\subsection{Model Derivation}

A simplified two dimensional block diagram of the neural connectivity proposed here is shown in figure (1-top) (for a three dimensional view of these series of neural layers, see figure (16-top)). Horizontal lines in figure (1-top) represent conductive grids. As shown in this figure, every neuron is connected by some dendrites to a conductive grid consisting of the interconnected dendrites of neurons of this layer. The axons of these neurons are then connected to the conductive grid of the next layer. The signals taken by dendrites in figure (1-top) from the conductive grid to a neuron could be summed together or subtracted before it is fed to a neuron. An example of a neural grid representing a single layer is shown in figure (1-bottom). In this figure, a black circle represents a neuron whose dendrites are connected to those of other neurons through the grid. It is important to note that the input of a single neuron is both directly connected to the output of one neuron in the previous layer (see figure (1-top)) and indirectly connected to the outputs of all other neurons in the previous layer through the conductive grid (see figure (1-bottom)). As explained later in this paper, the contribution of all indirectly connected neurons is mathematically determined by a convolution between the signals in their axons and the impulse response of the conductive grid. Let us now focus on one of the aforementioned layers of neurons. One portion of the conductive grid of a typical neural layer consisting of four neurons is shown in figure (2). As shown in this figure, dendritic potentials at layer $l$ represented by $u(x, y, t, l)$ are conducted through resistors, and capacitors. In this figure, voltages $v(x, y, t, l-1)$ are assumed to come from dendritic spine head voltages taken from (the axons of neurons of) the previous layer 
(layer $l-1)$. The model parameters are as follows:

$R_{x l}$ and $R_{y l}=$ cytoplasmic resistivity of dendrite per unit length for layer $l$

$C_{x l}$ and $C_{y l}=$ membrane capacities per unit length for layer $l$

$G_{x l}$ and $G_{y l}=$ conductance across dendritic membrane in unit length for layer $l$

As shown in this figure, spine heads in layer $l$ are modelled as electrical amplifiers with high input impedances, low output impedances and gains of $A_{l}$. More details of such amplifiers are shown in figure (3). It is reported in the literature (see e.g. (Miller et al. 1985; Segev and Rall 1988)) that spine heads are active circuits and amplify spikes. This behaviour of spine heads guarantees that the transmission of spikes is maintained through all layers in the biological visual pathway. Therefore in steady state, the visual signal is passed through spine heads with no attenuation.

As a result, the steady state of spine heads is modelled as an electrical amplifier. Before we write the equations for the two dimensional transmission grid, let us write the relationship between $v(x, y, Z, t, l): R^{3} \times R^{+} \times N \rightarrow R$ and $u(x, y, t, l): R^{2} \times R^{+} \times N \rightarrow R$ (see figure (3)) by using the model of an electrical amplifier for spine heads, where $\mathrm{Z}$ is the length of the axon connecting two consecutive layers at location $(x, y)$ in the visual field. By considering the Kirchhoff Voltage Law (KVL) in left and right loops in figure (3), we can write:

$i_{o}=\frac{u(x, y, t, l)-A_{l} v(x, y, Z, t, l)}{R_{o l}}$

where $i_{o}$ and $R_{o l}$ are the output current going into the transmission grid per unit length and the output resistance of the amplifier.

Let us now consider the Kirchhoff Current Law (KCL) for linear circuits in the node with the voltage $u(x, y, t, l)$ in figure (2):

$i_{x}(x, y, t, l)=G_{x t} \Delta x u(x, y, t, l)+i_{x}(x+\Delta x, y, t, l)+C_{x l} \Delta x \frac{\partial i_{x}}{\partial t}+i_{o x} \Delta x$ 
$i_{y}(x, y, t, l)=G_{y l} \Delta y u(x, y, t, l)+i_{y}(x, y+\Delta y, t, l)+C_{y l} \Delta y \frac{\partial i_{y}}{\partial t}+i_{o y} \Delta y$

where $t, i_{o x}$ and $i_{o y}$ are time, $x$ and $y$ components of the output current $i_{o}$ respectively. As shown in figures (1-bottom) and (2), $\Delta x$ and $\Delta y$ are small distances between two neighbouring neurons in $x$ and $y$ directions. $C_{x l}$ and $C_{y l}$ are considered very small and hence ignored here, therefore equations (2) and (3) can be written as two difference equations, i.e.:

$$
\begin{aligned}
& i_{x}(x, y, t, l)-i_{x}(x+\Delta x, y, t, l)=\left(G_{x l} u+i_{o x}\right) \Delta x \\
& i_{y}(x, y, t, l)-i_{y}(x, y+\Delta y, t, l)=\left(G_{y l} u+i_{o y}\right) \Delta y
\end{aligned}
$$

We also write KVLs between nodes with voltages $u(x, y, t, l)$ and $u(x+\Delta x, y, t, l)$ as well as $u(x, y, t, l)$ and $u(x, y+\Delta y, t, l):$

$$
\begin{aligned}
& u(x, y, t, l)-u(x+\Delta x, y, t, l)=R_{x l} \Delta x i_{x} \\
& u(x, y, t, l)-u(x, y+\Delta y, t, l)=R_{y l} \Delta y i_{y}
\end{aligned}
$$

To consider boundary conditions, let us assume that the boundary coincide with a line parallel to $y$ axis. In this case, on the boundary $i_{x}=0$ and equation (6) is simplified as:

$$
u(x, y, t, l)-u(x+\Delta x, y, t, l)=0
$$

Similarly for a boundary parallel to $x$ axis, the boundary condition is:

$$
u(x, y, t, l)-u(x, y+\Delta y, t, l)=0
$$

In general, the boundary condition for a boundary with any orientation is $u(x, y, t, l)-u(x+\Delta x \cos (\theta), y+\Delta y \sin (\theta), t, l)=0 \quad$ (Neumann boundary condition), where $\tan (\theta)=\frac{n_{y}}{n_{x}}$ and $\vec{n}=n_{x} \vec{i}+n_{y} \vec{j}$ is the normal unit vector to the boundary.

Due to the rotational symmetry (our third assumption) in the conductive grid with respect to $x$ and $y$ axes in figure (2), we assume $\Delta x=\Delta y=\Delta, G_{x l}=G_{y l}=G_{l}, \quad R_{x l}=R_{y l}=R_{l}$ and 
$i_{o x}=i_{o y}=\frac{i_{o}}{2}$. Therefore from equations (1), (4), and (5), we can write the following difference equation:

$$
\begin{aligned}
& u(x+\Delta x, y, t, l)-2 u(x, y, t, l)+u(x-\Delta x, y, t, l)+u(x, y+\Delta y, t, l)-2 u(x, y, t, l)+u(x, y-\Delta y, t, l) \\
& -\Delta^{2}\left(2 R_{l} G_{l}+\frac{R_{l}}{R_{o l}}\right) u(x, y, t, l)=-\frac{\Delta^{2} R_{l} A_{l} v(x, y, Z, t, l)}{R_{o l}}
\end{aligned}
$$

Let us now take a two dimensional $\mathrm{Z}$ transform from both sides of difference equation (10) to calculate the transfer function $\frac{U\left(z_{x}, z_{y}, t, l\right)}{V\left(z_{x}, z_{y}, Z, t, l\right)}$, i.e.:

$$
H_{\Delta}\left(z_{x}, z_{y}\right)=\frac{U\left(z_{x}, z_{y}, t, l\right)}{V\left(z_{x}, z_{y}, Z, t, l\right)}=\frac{-\frac{R_{l} A_{l} \Delta^{2}}{R_{o l}}}{z_{x}-2+z_{x}^{-1}+z_{y}-2+z_{y}^{-1}-\left(2 R_{l} G_{l}+\frac{R_{l}}{R_{o l}}\right) \Delta^{2}}
$$

The transfer function $H_{\Delta}\left(z_{x}, z_{y}\right)$ with respect to radian frequencies $\Omega_{x}, \Omega_{y} \in[-\pi, \pi)$ is written as:

$$
H_{\Delta}\left(e^{j \Omega_{x}}, e^{j \Omega_{y}}\right)=\frac{-\frac{R_{l} A_{l} \Delta^{2}}{R_{o l}}}{e^{j \Omega_{x}}-2+e^{-j \Omega_{x}}+e^{j \Omega_{y}}-2+e^{-j \Omega_{y}}-\left(2 R_{l} G_{l}+\frac{R_{l}}{R_{o l}}\right) \Delta^{2}}
$$

Or

$$
H_{\Delta}\left(e^{j \Omega_{x}}, e^{j \Omega_{y}}\right)=\frac{-\frac{R_{l} A_{l} \Delta^{2}}{R_{o l}}}{2\left(\cos \left(\Omega_{x}\right)-1\right)+2\left(\cos \left(\Omega_{y}\right)-1\right)-\left(2 R_{l} G_{l}+\frac{R_{l}}{R_{o l}}\right) \Delta^{2}}
$$

In figure (4), the transfer function $H$ is plotted for $\Omega_{x} \in[-\pi, \pi), \quad \Omega_{y}=0$, $\frac{R_{l} A_{l}}{R_{o l}}=2 R_{l} G_{l}+\frac{R_{l}}{R_{o l}}=1$ and $\Delta=0.01,0.1$, and 1. As shown in this figure, the smaller the value of $\Delta$, the closer the transfer function $H$ gets to the Fourier transform of a modified Bessel function of the second kind and zero degree. In the following theorem, we prove that 
the transfer function $H_{\Delta}\left(e^{j \Omega_{x}}, e^{j \Omega_{y}}\right)$ approaches the Fourier transform of a modified Bessel function of the second kind and zero degree, as $\Delta \rightarrow 0$.

Theorem 1: The transfer function $H_{\Delta}\left(e^{j \Omega_{x}}, e^{j \Omega_{y}}\right)$ of equation (13) approaches the Fourier transform of a modified Bessel function of the second kind and zero degree as $\Delta \rightarrow 0$.

The proof of this theorem is presented in Appendix A.

According to one of our aforementioned assumptions (assumption 4), the distance between two neighbouring neurons is negligible in comparison with the dimensions of the visual field, i.e. $\Delta \rightarrow 0$. The result of theorem 1 (see equation (A-4)) indicates that the transfer function $H_{\Delta}$ with respect to frequencies $\omega_{x}$ and $\omega_{y}$ of continuous two dimensional visual signals can be written as the Fourier transform of a modified Bessel function of the second kind and zero degree (Mahmoodi 2012; Mumford and Shah 1989), if $\Delta \rightarrow 0$, i.e.:

$$
\frac{U\left(\omega_{x}, \omega_{y}, t, l\right)}{V\left(\omega_{x}, \omega_{y}, Z, t, l\right)}=\frac{A_{l} \frac{R_{l}}{R_{o l}}}{\omega_{x}^{2}+\omega_{y}^{2}+2 R_{l} G_{l}+\frac{R_{l}}{R_{o l}}}=\frac{a_{l}}{\omega_{x}^{2}+\omega_{y}^{2}+b_{l}}
$$

where $a_{l}=A_{l} \frac{R_{l}}{R_{o l}}$ and $b_{l}=2 R_{l} G_{l}+\frac{R_{l}}{R_{o l}}$ are the parameters associated with layer $l$. By $\Delta$ approaching zero, the conductive grid approaches to a conductive sheet. This conductive sheet corresponding to the $l^{\text {th }}$ layer whose transfer function with respect to the coordinates $x$ and $y$ of the retina's visual field is given in equation (14), is a model for the dendrites of all neurons of the $l^{\text {th }}$ layer. These neurons are also connected to the dendrites (modelled by another conductive sheet) of the next layer of neurons through their axons as shown in figure (1-top). Let us now model the axons with a transmission line along the axis $\mathrm{z}$ as shown in figure (5). The differential equation describing this transmission line can be written as:

$$
R_{z} C_{z} \frac{\partial v}{\partial t}+R_{z} G_{z} v-\frac{\partial^{2} v}{\partial z^{2}}=0
$$


where $v: R^{3} \times R^{+} \times N \rightarrow R$ is the potential of the axon membrane and

$R_{z}=$ cytoplasmic resistivity of axon per unit length

$C_{z}=$ membrane capacities of axon per unit length

$G_{z}=$ conductance across axon membrane per unit length

The impulse response of the axon can then be calculated by solving equation (15) for

$\delta(t)$ as the input to provide the impulse response $v_{h}: R \times R^{+} \rightarrow R$ as follows:

$v_{h}(z, t)=\frac{1}{2} \sqrt{\frac{1}{\pi t R_{z} C_{z}}} \exp \left(-\frac{G_{z} t}{C_{z}}\right) \exp \left(-\frac{R_{z} C_{z} z^{2}}{4 t}\right)$

In equation (16), $z$ could be interpreted as the time scale parameter of the time smoothing kernel. Equation (16) in spatial frequency domain is also written as:

$V_{h}\left(\omega_{z}, t\right)=\frac{1}{R_{z} C_{z}} \exp \left(-\frac{G_{z} t}{C_{z}}\right) \exp \left(-\frac{\omega_{z}^{2} t}{R_{z} C_{z}}\right)$

where $\omega_{z}$ is the spatial frequency corresponding to the spatial variable $z$. Equation (17) is similar to the temporal discrete model with the truncated exponential first-order integrators in a work done simultaneously and independently in (Lindeberg 2015). From equation (17), it is therefore concluded that neural axons behaving like transmission lines are the electrical circuitries in biological vision to provide the exponential first order kernels (integrators) proposed in (Lindeberg 2013, Lindeberg 2015). In fact in our model, the serially coupled first order kernels discussed in (Lindeberg 2015) are achieved by considering the fact that the neural layers and therefore their axons are cascaded. However the difference between our work discussed here and the work presented in (Lindeberg 2015) is that the motivation behind equation (17) is the anatomy and structure of biological visual systems in vertebrate and the way the neurons are spatially arranged to transmit the visual signal from retina to visual cortex, whilst the inspiration for the cascaded first order integrators proposed in (Lindeberg 2015) is to 
investigate time-causal spatio-temporal receptive fields with discrete temporal scale levels.

\subsection{Neural Spikes}

It is well known that a neuron transmit a signal by sending spikes through its axon to another neuron. In our model, the neurons of one layer send spikes to those of the next layer to transmit visual signals to other layers towards the visual cortex. However no spike is exchanged among the neurons within the same layer, since the neurons associated with a certain layer are connected through dendrites and not axons. In this paper, we exploit the simple and classic rectification model in which firing rate is zero for membrane potentials below a certain threshold and grows linearly with membrane potentials over the threshold (e.g. see (Granit et al. 1963; Carandini and Fester 2000)). Here, we therefore demonstrate how a nonlinear system of neurons communicating with spikes through axons can be approximated as a linear system for low pass signal transmission under a certain condition.

Since axons behave like a transmission line, spikes modelled here as Dirac delta functions (impulses) need to be transmitted via axons. As a result, the output signal at the end of the transmission line (axon) has the general form of temporal impulse response of the line (axon) for a single spike (impulse) as derived in equation (16). This temporal impulse response for some certain values of the parameters is shown in figure (11-top row-left column). As can be seen from this figure and equation (16), this temporal impulse response is a low pass signal. This implies that the axon modelled as a transmission line behaves like a low pass filter. Such a low pass filter removes medium to high frequency components of the train of spikes (impulses) generated by the neuron in the previous layer, so that the signal reached to the dendrites of the next layer is a low pass signal whose maximum amplitude is proportional to the number of spikes (impulses) generated by the neuron of the previous layer. This low pass signal is also affected by the low pass signals reached to the neighbouring neurons, so that the 
low pass signals reached to neurons in this neighbourhood are also filtered by low pass filter (14). Then a particular neuron in this layer fires spikes according to the potential (low pass signal) it senses in its dendrites. In theorem 2, under some certain condition, we demonstrate that the number of spikes a neuron fires in a given layer depends on the number of spikes it receives through its dendrites. In the following theorem, $\bar{T}$ and $\sigma$ are defined as the average and standard deviation of the time intervals between consecutive spikes in a train of spikes sent by a neuron through its axon so that the average firing rate of a train of spikes is $\frac{1}{\bar{T}}$.

Theorem 2: if neuron A sends a train of $N$ spikes with an average $\bar{T}$ and standard deviation $\sigma$ of the time intervals of its train of spikes for a certain static visual signal through its axon to neuron $\mathrm{B}$, the potential sensed by neuron $\mathrm{B}$ in its dendrites is proportional to $N$, provided that all time intervals between two consecutive spikes is too small with respect to the time $(t)$ taken for the signal to reach neuron B.

The proof is presented in Appendix B.

According to the result of theorem 2 , the axon of a neuron in layer $l-1$ is connected to a neuron in layer $l$, if the neuron in layer $l-l$ fires $N$ spikes, the potential on the neuron at the end of the neuron's axon in layer $l$ will be proportional to $N$ (see figure (5)), i.e.:

$$
v(x, y, z, t, l)=v_{h}(z, t) N\left(1+(2 a+1) \bar{T}+a \sigma^{2}\right)
$$

where $v_{h}(z, t)$ is given in equation (16) and $a=\frac{G_{z}}{C_{z}}-\frac{R_{z} C_{z} z^{2}}{4 t^{2}}$.

On the other hand, according to classical rectification model for neural firing rates (Granit et al. 1963; Carandini and Fester 2000), if $u(x, y, t, l-1)$ is greater a certain threshold, then $u(x, y, t, l-1)=K N$

where $K$ is a constant. From equations (18) and (19), it is therefore easy to see that potential $u(x, y, t, l-1)$ is linearly proportional to potential $v(x, y, z, t, l)$, i.e.: 
$v(x, y, z, t, l)=k v_{h}(z, t) u(x, y, t, l-1)$

where $k$ is also a constant.

The limit between linearity and nonlinearity of the visual system in our model depends on how small or large the time intervals between consecutive spikes is with respect to the time that neural spikes take to reach to the next layer from the current layer. If these time intervals are large enough then relations between the input potentials of a layer with the input potentials of the next layer will become nonlinear. In such cases the whole visual system behaves like a nonlinear system.

By using equations (14), (17), and (20), the transfer function of a neuron whose dendrites and axon are connected to the conductive sheets of layer $l-1$ and layer $l$ respectively with respect to spatial frequencies $\omega_{x}, \omega_{y}, \omega_{z}$ and time $t$ is written as:

$$
L\left(\omega_{x}, \omega_{y}, \omega_{z}, t, l\right)=\frac{U\left(\omega_{x}, \omega_{y}, t, l\right)}{U\left(\omega_{x}, \omega_{y}, t, l-1\right)}=\frac{a_{l}}{R_{z} C_{z}\left(\omega_{x}^{2}+\omega_{y}^{2}+b_{l}\right)} \exp \left(-\frac{G_{z} t}{C_{z}}\right) \exp \left(-\frac{\omega_{z}^{2} t}{R_{z} C_{z}}\right)
$$

Let us now assume that the visual path consists of $n$ cascaded layers whose transfer functions are given by $L\left(\omega_{x}, \omega_{y}, \omega_{z}, t, l\right)$ in the above equation. The transfer function of $n$ cascaded layers denoted by $L_{1: n}$ can therefore be written in equation (21).

$$
L_{1: n}\left(\omega_{x}, \omega_{y}, \omega_{z}, t\right)=\frac{t^{n-1}}{\left(R_{z} C_{z}\right)^{n} \Gamma(n)} \exp \left(-\frac{G_{z} t}{C_{z}}\right) \exp \left(-\frac{\omega_{z}^{2} t}{R_{z} C_{z}}\right) \prod_{t=1}^{n}\left(\frac{a_{l}}{\omega_{x}^{2}+\omega_{y}^{2}+b_{l}}\right)
$$

where $\Gamma$ is the gamma function. Our assumption in (21) is that the axons of different layers have the same parameters $C_{z}, R_{z}$ and $G_{z}$. However it is straightforward to verify that even if the axons of different layers have different line parameters, transfer function (21) will still have a similar form but with different parameters in exponential terms. It is noted that the first part of transfer function (21) is due to $n$ cascaded axons which are equivalent to the $n$ serially coupled temporal first order kernels proposed in (Lindeberg 2015). 
In theorem 3, we prove that if the number of cascaded layers approaches infinity, the Green function of the system of $n$ cascaded neural layers approaches a Gaussian function with respect to $x, y$, and $z$.

Theorem 3: For $\forall l \in \mathrm{N} \quad b_{l}>1$ where $\mathrm{N}$ is the set of the Natural numbers, the transfer function in (21) approaches a Gaussian transfer function as $n$ (the number of cascaded layers) approaches infinity.

A proof for theorem 3 is presented in Appendix C.

The similarities and differences between theorem 3 and Tikhonov regularization (Nielsen et al. 1997) are interesting. In this paper, the Gaussian kernel is resulted from two facts: 1) a single layer of neurons behaves like a conductive sheet and 2) neural layers are cascaded from retina to visual cortex to transmit the visual signal from eyes to brain. However no regularization, or optimisation is employed here to derive the Gaussian kernel. On the other hand, the Gaussian kernel derived from Tikhonov regularization is the result of the optimization of an energy functional (Nielsen et al. 1997). In this regularization framework, further constraints such as scale invariance, semi-group and non-negativity properties are also required to result in a Gaussian kernel as the optimal solution of the Tikhonov optimization problem. It is also interesting to note that the constraint of semi-group property in Tikhonov regularization can be considered equivalent to the assumption of cascaded layers in our model. Further, the transfer function derived in (14) already enjoys the scale-invariance and non-negativity properties. In other words, scale-invariance and non-negativity properties are inherent in our model due to the electrical (physical) properties of conductive layers.

By taking spatial inverse Fourier transform from equation (C-9) with respect to $x, y$, and $z$ and their spatial frequencies, transfer function (21) in the spatio-temporal domain for a large $n$ can be written as: 
$h(x, y, z, t)=\mathcal{F}^{-1}\left(L_{1: n}\left(\omega_{x}, \omega_{y}, \omega_{z}, t\right)\right)=\frac{A b_{i}^{2}}{(4 \pi n)^{3 / 2}} \psi_{n}(z, t) K_{n}(x, y)$

where $\mathcal{f}^{-l}$ is the three dimensional inverse Fourier transform and

$$
\begin{aligned}
& \psi_{n}(z, t)=\frac{t^{\frac{n}{2}-1}}{\sqrt{\left(R_{z} G_{z}\right)^{n}}} \exp \left(-\frac{G_{z} t}{C_{z}}\right) \exp \left(-\frac{R_{z} C_{z} z^{2}}{2 t}\right) \\
& K_{n}(x, y)=\underbrace{K(x, y)^{*} K(x, y) * \ldots * K(x, y)}_{n \text { times }}
\end{aligned}
$$

And $A$ is a constant and defined in Appendix $\mathrm{C}$ as:

$$
A=\frac{\prod_{l=1}^{n} a_{l}}{\left(1+\sum_{k=1}^{P} f_{k}\left(\varepsilon_{1}, \varepsilon_{2}, \ldots, \varepsilon_{n}\right)\right.}
$$

$K(x, y)$ is also the modified Bessel function of the second type and zero degree.

\subsection{Some Numerical Considerations for Our Model}

We notice that for small values of $n, K_{n}(x, y)$ is the repeated convolutions of $n$ modified Bessel functions of the second kind and zero degree. However according to theorem 3 for large values of $n, K_{n}(x, y) \sim \frac{b_{i}}{n} \exp \left(-\frac{b_{i}}{n}\left(x^{2}+y^{2}\right)\right)$. The result of theorem 3 is in complete agreement with the biological recordings of retinal cells (see e.g. (Enroth-Cugell and Robson 1966; Young 1987)).

In practise, as low as six consecutive layers of neurons can result in a kernel very close to a Gaussian kernel. Here, we attempt to show that $K_{n}(x, y)$ in $(22)$ is close to a Gaussian kernel even with a limited number of layers. The kernel obtained from the output of the $l^{\text {th }}$ layer is compared with an equivalent Gaussian kernel associated with the corresponding layer in frequency domain in figure (6). In this experiment, we allow the parameter $b_{l}$ in equation (21) for each layer to vary slightly in comparison with other layers by sampling its values 
from a Gaussian distribution with a certain mean and standard deviation (in this experiment, the mean value and standard deviation are chosen as unity and 0.2 respectively). A varying parameter $b_{l}$ indicates that the neural layers are not identical however they have similar electrical structures. In figure (6), a cross section of the kernels are plotted for illustration purposes and the comparison has been made for layers $l=1,2,3,4,5$ and 6 . As shown in this figure, the difference between the two kernels for the first layer is significant. However as $l$ increases, the difference between the two filters becomes lower and lower so that for the $6^{\text {th }}$ layer, the difference between the two filters is almost negligible. In this figure, the kernel obtained from the network of neurons is plotted with the dotted curve and the Gaussian kernel for the corresponding layer is drawn with the dashed curve.

The Euclidean distance between the two kernels in frequency domain for various values of layers between unity and one hundred is also plotted in figure (7). As can be seen from this figure, the differences between the two kernels are negligible when the number of layers, $l$, is more than six. In this experiment, $b_{l}$ is again allowed to vary slightly from a layer to the next one according to a Gaussian distribution with a unity mean and a standard deviation of 0.1 . In the next experiment, the spatial part of the filter derived in equation (21) is implemented for six layers and the case where $a_{l}=b_{l}$ is sampled from a Gaussian distribution with the mean and standard deviation of unity and 0.1 respectively. The results are shown in figure (8). Figure (8-left) depicts the original image and the filtered image calculated by using kernel (21) is shown in figure (8-middle). The image filtered by the equivalent Gaussian kernel of equation (C-9) with $n=6$ is also presented in figure (8-right). The difference between the two filtered images per pixel for the first ten layers is plotted in figure (9), for the case where $a_{l}$ and $b_{l}$ randomly vary from a layer to the next, as considered in the previous experiments. 
As can be seen from these figures, by increasing the number of layers, the image filtered by using transfer function (21) approaches an image filtered by a Gaussian kernel given in (C-9) with $n$ equal to the number of layers.

In figure (10), we compare the Laplacian of Gaussian (figure (10-left)) with the Laplacian of spatial kernel $K_{n}(x, y)$ for $n=6$ (six neural layers) as shown in figure (10-middle). As reported in (DeAngelis et al. 1995), an example of a biologically recorded spatial receptive field profile of an LGN cell is also presented in figure (10-right).

Figure (11) depicts the time casual kernel (impulse response) computed by using equation (16) and its regular first and second derivatives. For comparison, the time causal kernel proposed by Lindeberg in (Lindeberg 2011; Lindeberg 2013) and its regular first and second derivatives are also shown in this figure. This figure demonstrates the resemblance of the time causal kernel calculated here by modelling the neural axons based on a transmission line with the time causal kernel proposed in (Lindeberg 2011; Lindeberg 2013). The first and second derivatives with respect to logarithmic and power law time transformation are also presented in figures (12) and (13) respectively. As can be seen from these figures, the first regular derivative of the time causal kernel calculated here has two peaks and one interior zero crossing. This is similar to the first regular derivative of the time causal kernel proposed in (Lindeberg 2011; Lindeberg 2013). It is also noted that for the first temporal derivatives of both kernels, the first peak is the strongest similar to the biologically recorded temporal response of "non-lagged cells" in LGN as shown in figure (14-left) (DeAngelis et al. 1995). For the second regular and power law time transformed derivatives, the first peak is also dominant in both time causal kernels, whereas the second peak in both kernels is strongest in their second logarithmic time transformed derivatives similar to the biologically recorded response of "lagged cells" in LGN as shown in figure (14-right) (DeAngelis et al. 1995). Figure (15) also compares the time-causal spatio-temporal kernels proposed in (Lindeberg 
2011; Lindeberg 2013) and the time-causal spatio-temporal kernels derived here based on our electrical model of the visual pathway. The similarity of shapes of these kernels with each other and with the biologically recorded spatiotemporal receptive profiles of lagged and nonlagged LGN cells shown in figure (14) (DeAngelis et al. 1995), is noticeable and interesting. Our numerical results depicted in figure (15) are also confirmed by the numerical results presented in a more recent work by Lindeberg (see figure (2) in Lindeberg 2015).

\section{Structural Properties of our Neural Model}

As discussed in section 2, the model we propose here for the early visual pathway, consists of a series of neural layers with electrical properties behaving linearly. These neural layers are connected in series. Theorem 3 proved in section 2 is exploited here to describe such a network of neurons.

\subsection{Diffusion and Convolution}

For small values of $\Delta$, difference equation (10) is approximated by the following partial differential equation:

$$
\nabla^{2} u(x, y, t, l)=b_{l} u(x, y, t, l)-a_{l} v(x, y, Z, t, l)
$$

where $Z$ and $t$ are the length of the axon connecting two layers and the time after which the visual signal has reached the $l^{\text {th }}$ layer and $b_{l}=2 R_{l} G_{l}+\frac{R_{l}}{R_{o l}}$ and $a_{l}=A_{l} \frac{R_{l}}{R_{o l}}$.

By using equation (18), equation (25) is written as:

$$
\nabla^{2} u(x, y, t, l)=b_{l} u(x, y, t, l)-a_{l} \frac{u(x, y, l-1)}{2} \sqrt{\frac{1}{\pi t R_{z} G_{z}}} \exp \left(-\frac{G_{z} t}{C_{z}}\right) \exp \left(-\frac{R_{z} C_{z} Z^{2}}{4 t}\right)
$$

By assuming that the visual signal has reached layer $l$ at time $t$, let us denote $u(x, y, t, l)$ with $u(x, y, l)$. For the case where $a_{l} \sqrt{\frac{R_{z} G_{z}}{\pi t}} \exp \left(-\frac{G_{z} t}{C_{z}}\right) \exp \left(-\frac{R_{z} G_{z} Z^{2}}{4 t}\right)=b_{l}$, by assuming that 
$\Delta l=1$, equation (25) can be interpreted as a discrete-time version of a continuous diffusion equation, in which $l \in R^{+}$denotes the $l^{\text {th }}$ layer:

$$
\nabla^{2} u(x, y, l)=b_{l} \frac{\partial u(x, y, l)}{\partial l}
$$

It is important to notice that $l$ in equation (26) is the layer number which behaves similar to the scale parameter in scale space formulation and therefore equation (26) is in complete agreement with the scale space theory for ideal biological vision systems. From equation (26), we therefore conclude that the initial image on the retina is smoothed by using a diffusion type process as it is propagated through the cascaded neural layers. According to our model, this is how the convolution operation (with a Gaussian filter) is implemented in biological visual systems. The solution of equations (25) or (26) is a series of Gaussian kernels with various scales. These equations therefore indicate that a Gaussian kernel with a certain scale is associated with each layer and the scale of the Gaussian kernel increases as the visual signal propagates toward visual cortex. As a result, biological visual systems access a multi-scale Gaussian kernel measurements.

By using our model, it is therefore straightforward to explain that the convolution of the initial signal of the visual field on retina with a Gaussian kernel is due to the electrical properties of the transmission sheets associated with the neural layers and the fact that these layers are connected in series as explained in section 2.

\subsection{Agreement with Scale Space Theory}

The model presented here is based on four biological related hypotheses. We therefore need to verify if our model is in agreement with the Scale space theory. In this subsection, we demonstrate that our model represented by the equations derived in section 2 meets the scale space structural requirements of an idealized visual front end as described in (Lindeberg 2013). The requirements discussed here are associated with a) static image data over a spatial domain b) time-dependent image data over a spatio-temporal domain. 
a) Static image data over a spatial domain

For the static image data which are independent of time, the scale space structural requirements are of our model operating linearly as follows:

a-i) Linearity and Convolution Structure:

Equation (22) represents the Green function of the visual system characterized by our model when it behaves linearly. A time-independent form of equation (22) has the following general structure:

$h(x, y) \sim K_{n}(x, y)$

where $K_{n}(x, y)$ is defined in equation (24). When our system meets the linearity condition in theorem 2, it is straightforward to verify that this system is a linear one and it is easy to conclude from equations (10), (14) and (24) that $K_{n}(x, y)$ is a convolution transformation.

a-ii) Image Measurements at Different Scales

The time-independent Green function of our model for ideal biological visions is of the form of equation (27). With a large number of cascaded layers, $n, K_{n}(x, y)$ is approximated as a 2D Gaussian filter (see theorem 3 for more details), i.e.:

$$
K_{n}(x, y) \sim \frac{b_{i}^{2}}{4 \pi n} \exp \left(-\frac{b_{i}^{2}}{4 n}\left(x^{2}+y^{2}\right)\right)
$$

where $b_{i}$ is a model parameter defined in Appendix C. It is numerically demonstrated in section (2) (see figure (6) and the related text for further details) that even with $n$ as low as six layers, $K_{n}(x, y)$ is a good approximation of a Gaussian filter. If a new layer is cascaded to the aforementioned $n$ cascaded layers, according to theorem 3 , the system of $n+1$ cascaded layers has a Green function approximated as a Gaussian function, i.e.:

$$
K_{n+1}(x, y) \sim \frac{b_{i}^{2}}{4 \pi(n+1)} \exp \left(-\frac{b_{i}^{2}}{4(n+1)}\left(x^{2}+y^{2}\right)\right)
$$


The kernel related to the $(n+1)^{\text {th }}$ layer is a modified Bessel function of the second kind and zero degree. However since $n$ and therefore $n+1$ is a large number, then according theorem 3 , $K_{n+1}(x, y)$ is approximated as Gaussian kernel as written in equation (29). By comparing equations (28) and (29), it is easy to conclude that every layer in a system of cascaded layers can provide an image measurement for a scale proportional to the total number of previous cascaded layers. It is noted that this scaling parameter is positive because the number of layers is always a positive number. Our model of biological vision in this paper is characterized with discrete number of layers, i.e. $n \in \mathrm{N}$. However if one aims to extend this model for continuous variable $n \in R^{+}$(as investigated in (Lindeberg 2013)), then it is straightforward to see from equation (28) that $K_{n}(x, y)$ approaches to identity operation for small values of $n$, i.e.:

$\lim _{\mathrm{n} \rightarrow 0} K_{n}(x, y)=\delta(x, y) \quad$ for $\quad n \in R^{+}$

where $\delta(x, y)$ is a delta Dirac function.

a-iii) Semigroup and cascaded properties

For any $m \in \mathrm{N}$ and large values of $n \in \mathrm{N}$, it is straightforward to conclude from theorem 3 (similar to the discussion in $(a-i i))$ that

$$
K_{n+m}(x, y)=K_{n}(x, y) * K_{m}(x, y)
$$

Equation (30) is also correct for positive continuous real values of $m$ and $n$, i.e. for any $m, n \in R^{+}$(positive valued scales are fully investigated in (Lindeberg 2011; Lindeberg 2013)). Equation (30) indicates that filters $K_{n}(x, y)$ form a semigroup with respect to convolution. It is also easy to infer from (30) that the transformation (convolution) between two different layers $m_{1}$ and $n_{1}$ with $m_{1}>n_{1}$ and $m_{1}-n_{1}$ being a large positive value, obeys a cascaded property. Therefore our model of biological vision possesses a multi-scale representation as indicated in section 3.1. 
a-iv) Infinitesimal generator

Obviously for discrete values of neural layers used in our model, equation (25) is considered as an infinitesimal generator. For continuous values of neural layers on the other hand, equation (26) is regarded as infinitesimal generator.

a-v) Smoothing property: non-enhancement of local exterma

Given that infinitesimal generator of transformed (convolved) visual signals is diffusion equation (26) and following theorems 6 in (Lindeberg 2011), it is by sufficiency implied that $K_{n}(x, y)$ (even for small values of $n$ ) satisfying diffusion equation (26) possesses the nonenhancement of local exterma. It is also straightforward to prove directly (without theorem 6 in (Lindeberg 2011)) that kernels $K_{n}(x, y)$ possess the property of the non-enhancement of local exterma.

a-vi) Rotational Symmetry

It is clear that $K_{n}(x, y)$ for any $n \in \mathrm{N}$ is a rotational symmetric filter.

b) Time-dependent image data over a spatio-temporal domain

Let us finally consider the spatial image data changing over time. The most important requirement for a time dependent image data belonging to a biological visual system is time causality. From equation (16) and figure (11-top row), it is obvious that the time impulse response of our model of an ideal biological vision system is time causal. It is also noted that parameter $z$ in equation (16) can be interpreted as time scaling parameter of our time smoothing kernel.

\subsection{Elongated Non-Isotropic Receptive Fields}

Figure (16-top) depicts a three dimensional view of the cascaded neural layers proposed here. Vertical planes in this figure correspond to neural layers. Figure (16-middle) shows the neural configuration of one of the vertical planes of figure (16-top). The network of neurons 
depicted in this figure represents a neural layer covering the visual field with $x$ and $y$ coordinates.

The spatial response of a neuron summing three neurons shown in red in figure (16-middle) produces non-isotropic elongated (affine Gaussian) kernels. As an example, the spatial impulse response of these three neurons can be written as:

$$
h_{n}(x, y)=\left(\left(K_{n}\right)_{x}(x, y-\Delta)+\left(K_{n}\right)_{x}(x, y)+\left(K_{x n}\right)_{x}(x, y+\Delta)\right)
$$

where $\Delta$ and $n$ are the distance between two neighbouring neurons in figure (16-middle) and $n$ is the layer number of the neural layer shown in figure (16-middle). Function $\left(K_{n}\right)_{x}(x, y)$ in (31) is the first derivative (with respect to $x$ ) of the $n$ repeated convolutions of the modified Bessel function of the second type and zero degree. Such a response is numerically simulated and shown in figure (17-left). The directional derivative (along $x$ direction) of the spatial response of a Gaussian kernel with $\frac{\sigma_{y}}{\sigma_{x}}=3$ is also depicted in figure (17-middle). An example of the biologically recorded spatial receptive profile of a simple cell with strong directional preference is also shown in figure (17-right) (DeAngelis et al. 1995). The similarity between figures (17-left) and (17-right) is noticeable. The spatio-temporal response of such a separable neuron is also modelled by:

$$
\kappa(x, y, t)=h_{n}(x, y) v_{t t}(z, t)
$$

where $h_{n}(x, y)$ and $v(z, t)$ are given in equations (31) and (16). The 2D spatial response given by equation (32) for three different times is shown in figure (18-top). Our results show a close resemblance to the recorded data presented in (DeAngelis et al. 1995) as depicted in figure (18-bottom).

\subsection{Motion Selectivity of Simple Cells in Striate cortex}

It is well known that motion selectivity and perception is realized in visual cortex. In fact, most cortical neurons are quite sensitive to stimulus velocity (DeAngelis et al. 1995; 
DeAngelis and Anzai 2004). It is rather straightforward to extend our model discussed in previous sections to also include motion selectivity. Figure (16-bottom) shows a neural configuration placed on the horizontal plane of figure (16-top). Each horizontal line is a 1D representation of a neural layer shown as a vertical plane in figure (16-top) and the flow of visual signal is from the bottom of figure (16-bottom) to the top (or from the outside of the page to inside of it in figure (16-top)). In this figure, neural axons shown as vertical lines in figure (16-bottom) in a layer, are connected to the dendrites of another neuron in the same location of the visual field in the next layer. Each single neuron on such a network is characterized with isotropic kernels in space and separable kernels in space-time as discussed in previous sections. A linear combination of some of these neurons on a single layer also results in a neuron with a non-isotropic separable spatio-temporal response as demonstrated in section 3.3.

A neuron summing the spatio-temporal responses of some diagonal non-isotropic separable neurons (shown in red circles in figure (16-bottom)) in this neural configuration produces a velocity-adapted non-separable spatio-temporal response which motion selective neurons in visual cortex are associated with. By changing the slope of the configuration of these diagonal neurons (shown in blue circles in figure (16-bottom) as another example), the linear combination of these neurons (shown in blue colour) produces another non-separable spatiotemporal response adapted to a different velocity. The spatio-temporal $1+2 \mathrm{D}$ response produced by summing the response of these separable non-isotropic neurons can be written as:

$$
\kappa(x, y, t)=\left(h_{n-1}(x-\Delta, y) \Pi\left(\frac{2(t-\tau)}{\tau}+\frac{\tau}{2}\right)+h_{n}(x, y) \Pi\left(\frac{2 t}{\tau}+\frac{\tau}{2}\right)+h_{n+1}(x+\Delta, y) \Pi\left(\frac{2(t+\tau)}{\tau}+\frac{\tau}{2}\right)\right) v_{t t}(z, t)
$$

where $\Delta$ and $\tau$ are shift in $x$ direction and time delay between two neighbouring red neurons in figure (16-bottom). Further, $\Pi(t)$ is a rectangular function and $h_{n}(x, y)$ and $v_{t t}(z, t)$ are 
also calculated from equations (31) and (16) respectively. It is noted that the temporal impulse response produced by the axons of the neurons in layers $n-1, n$ and $n+1$ (i.e. the red neurons in figure (16-bottom)) in equation (23) are approximated as a rectangular function for simplicity and $v_{t t}(z, t)$ is the second derivative of temporal impulse response for the axon of the neuron (not shown in figure (16)) summing the red neurons in figure (16-bottom).

Figure (19-top) presents the simulated 1+2D spatio-temporal response of a velocity adapted non-isotopic neuron for three different times. The results shown in this figure closely resemble the recorded spatio-temporal receptive profile of a velocity adapted neuron reported in (DeAngelis et al. 1995) as shown in figure (19-bottom).

Figure (20-top) shows the spatio-temporal 1+1D responses, $h_{x x t}$ (see equation(22)) of three separable neurons such as the red neurons in figure (16-bottom) for $R_{z} C_{z}=1.9, \frac{G_{z}}{C_{z}}=0.01$, $R_{z} C_{z} z^{2}=400, \mu=400$ with layer numbers $n=10,11$ and 12 in equation (22). Our numerical results in figure (20-top) is closely similar to the recorded 1+1D spatiotemporal receptive profile of a separable neuron reported by DeAngelis et al. (1995), as presented in figure (20bottom). Figure (21-left) shows a velocity-adapted non-separable spatio-temporal 1+1D response produced by summing the three separable isotropic spatio-temporal $1+1 \mathrm{D}$ responses shown in figure (20-top). The velocity-adapted spatio-temporal response shown in figure (21left) closely resembles the spatio-temporal receptive profile recorded in (DeAngelis et al. 1995) as shown in figure (21-right). The spatio-temporal separable $1+1 \mathrm{D}$ responses, $h_{x t}$, of three simple cells in the striate cortex with a configuration similar to that shown in red in figure (16-bottom) is also depicted in figure (22-top). An example of a recorded spatiotemporal receptive profile is also depicted in figure (22-bottom) as reported in (DeAngelis et al. 1995) to show the similarity between the result of our model and the biologically recorded data. As shown in figure (23-left), a velocity-adapted non-separable 
spatio-temporal $1+1 \mathrm{D}$ response closely resembling the spatio-temporal receptive profile of a simple cell in the striate cortex as shown in figure (23-right) and reported in (DeAngelis et al. 1995 ) is produced by summing the three separable spatio-temporal $1+1 \mathrm{D}$ responses shown in figure (22-top). In a more recent and simultaneous work as ours, Lindeberg proposes serially coupled integrators for discrete time-causal smoothing kernels whose numerical results (see figure(2) in (Lindeberg 2015)) confirm our numerical results presented in figures (21) and (23).

\section{Conclusion}

Previous works in the literature demonstrate that Gaussian-related kernels are used in biological visual systems. In a mathematical setting, Gaussian related kernels are derived for universal visual systems in the Gaussian scale space theory. However no comprehensive model based on biological hypotheses for visual systems is presented to show how a set of neurons produces Gaussians-related kernels.

A plausible neural circuitry matching an ideal visual front end of a biological visual system based on distributed electrical circuits is proposed here to model receptive fields in linear cells. In other words, this study presents a more detailed view of a plausible hardware for an ideal front end of a biological visual system. We have analytically demonstrated that our model behaves linearly under some certain condition. We have also explored the properties of our hardware model under the linear condition. Fundamental distributed electrical equations for conductive grids lead to a suggestion that an ideal visual front end of a biological visual system forms a kernel approximating a Gaussian kernel and its derivatives to process images taken from sensory cells in retina.

Our formulation also demonstrates that convolution with Gaussian kernels is implemented by using a diffusion equation as visual signals are propagated through cascaded layers of neurons in a biological visual system. Such cascaded layers therefore provide image 
measurements at various scales. Here it is demonstrated that cascaded neural layers with the transfer functions of the type of a modified Bessel function of the second kind and zero degree is equivalent to a Gaussian kernel with a certain standard deviation proportional to the number of cascaded layers. A model combining some isotropic cells, is also presented here to produce the spatial response of non-isotopic simple cells. Separable and non-separable (velocity adapted) non-isotropic simple cells are also successfully modelled here. Our numerical results obtained from our model are in complete agreement with both recorded biological data and numerical results obtained from the scale space theory.

For future work, it is interesting to extend this framework to explain the behaviour of nonlinear cells by employing nonlinear electrical elements as well as by exploring the properties of our proposed visual system under nonlinear conditions. Another interesting question to be addressed as a future work is that whether it is possible to model higher level visual algorithms such as segmentation and recognition implemented by biological vision systems by considering neural connectivities as linear/nonlinear electrical circuits.

\section{Appendices:}

\section{Appendix A: Proof of theorem 1}

Proof: Let us write transfer function (13) as:

$$
H_{\Delta}\left(e^{j \Omega_{x}}, e^{j \Omega_{y}}\right)=\frac{-\frac{R_{l} A_{l}}{R_{o l}}}{\frac{2\left(\cos \left(\Omega_{x}\right)-1\right)}{\Delta^{2}}+\frac{2\left(\cos \left(\Omega_{y}\right)-1\right)}{\Delta^{2}}-\left(2 R_{l} G_{l}+\frac{R_{l}}{R_{o l}}\right)}
$$

The transfer function $H_{\Delta}$ approaches zero for any $\Omega_{x} \neq 0$ or $\Omega_{y} \neq 0$ as $\Delta \rightarrow 0$. Because as $\Delta \rightarrow 0$, the term $\frac{\left(\cos \left(\Omega_{x}\right)-1\right)}{\Delta^{2}}$ in the denominator of transfer function (A-1) approaches infinity for any $\Omega_{x} \neq 0$. This is also true for the term $\frac{\left(\cos \left(\Omega_{y}\right)-1\right)}{\Delta^{2}}$. However for the values 
of both $\Omega_{x}$ and $\Omega_{y}$ very close to zero, the terms $\frac{\left(\cos \left(\Omega_{x}\right)-1\right)}{\Delta^{2}}$ and $\frac{\left(\cos \left(\Omega_{y}\right)-1\right)}{\Delta^{2}}$ in (A-1) become undetermined. To evaluate these terms for small values of $\Omega_{x}, \Omega_{y}$ and $\Delta$, we expand $\cos \left(\Omega_{x}\right)$ and $\cos \left(\Omega_{y}\right)$ with respect to $\Omega_{x}$ and $\Omega_{y}$ respectively by using the Taylor's series. Therefore the terms $\frac{\left(\cos \left(\Omega_{x}\right)-1\right)}{\Delta^{2}}$ and $\frac{\left(\cos \left(\Omega_{y}\right)-1\right)}{\Delta^{2}}$ are written as:

$$
\begin{aligned}
& \frac{\left(\cos \left(\Omega_{x}\right)-1\right)}{\Delta^{2}}=-\frac{\Omega_{x}^{2}}{2 \Delta^{2}}+\frac{\Omega_{x}^{4}}{24 \Delta^{2}}-\ldots \\
& \frac{\left(\cos \left(\Omega_{y}\right)-1\right)}{\Delta^{2}}=-\frac{\Omega_{y}^{2}}{2 \Delta^{2}}+\frac{\Omega_{y}^{4}}{24 \Delta^{2}}-\ldots
\end{aligned}
$$

Since $\Omega_{x}$ and $\Omega_{y}$ are very close to zero, we can ignore terms $\frac{\Omega_{x}^{4}}{24 \Delta^{2}}$ and $\frac{\Omega_{y}^{4}}{24 \Delta^{2}}$ and the higher orders of $\Omega_{x}$ and $\Omega_{y}$ in (A-2) and (A-3). Therefore the transfer function (A-1) is approximated as:

$\lim H_{\Delta}\left(e_{\Delta \rightarrow 0}^{j \Omega_{x}}, e^{j \Omega_{y}}\right)=\frac{-\frac{R_{l} A_{l}}{R_{o l}}}{-\frac{\Omega_{x}^{2}}{\Delta^{2}}-\frac{\Omega_{y}^{2}}{\Delta^{2}}-\left(2 R_{l} G_{l}+\frac{R_{l}}{R_{o l}}\right)}=\frac{\frac{R_{l} A_{l}}{R_{o l}}}{\omega_{x}^{2}+\omega_{y}^{2}+\left(2 R_{l} G_{l}+\frac{R_{l}}{R_{o l}}\right)}$

where $\omega_{x}=\frac{\Omega_{x}}{\Delta}$ and $\omega_{y}=\frac{\Omega_{y}}{\Delta}$ are spatial frequencies of continuous signals, i.e. in $\mathrm{rad} /(\mathrm{unit}$ length). Transfer function (A-4) is the Fourier transform of a modified Bessel function of the second kind and zero degree.

\section{Appendix B: Proof of theorem 2}

Proof: Neuron A sends a train of spikes (Dirac delta functions) through its axon to Neuron B by placing this train of spikes on its axon (a transmission line). Therefore the signal on the input of the transmission line is: 
$w_{i}(t)=\sum_{n=1}^{N} \delta\left(t-T_{n}\right)$

The impulse response of this axon with length $z$, is given in equation (16). Therefore the response of the transmission line (axon) to this train of spikes at $\mathrm{z}$ is as follows:

$w_{o}(z, t)=\sum_{n=1}^{N} v_{h}\left(z, t-T_{n}\right)$

where $v_{h}(z, t)$ is given in (16).

Let us now consider the response of the transmission line (axon) to the single spike $\delta\left(t-T_{n}\right)$ at $z$ :

$v_{h}\left(z, t-T_{n}\right)=\frac{1}{2} \sqrt{\frac{1}{\pi\left(t-T_{n}\right) R_{z} C_{z}}} \exp \left(-\frac{G_{z}\left(t-T_{n}\right)}{C_{z}}\right) \exp \left(-\frac{R_{z} C_{z} z^{2}}{4\left(t-T_{n}\right)}\right)$

Given that $T_{n}$ is too small, $v_{h}\left(z, t-T_{n}\right)$ is approximated as:

$v_{h}\left(z, t-T_{n}\right)=\frac{1}{2} \sqrt{\frac{1}{\pi t R_{z} C_{z}}}\left(1+\frac{T_{n}}{2 t}\right) \exp \left(-\frac{G_{z}\left(t-T_{n}\right)}{C_{z}}\right) \exp \left(-\frac{R_{z} C_{z} z^{2}}{4 t}\left(1+\frac{T_{n}}{t}\right)\right)$

By re-arranging the above equation, one can write:

$v_{h}\left(z, t-T_{n}\right)=v_{h}(z, t) \exp \left(a T_{n}\right)+\frac{T_{n}}{2 t} v_{h}(z, t) \exp \left(a T_{n}\right)$

where $a=\frac{G_{z}}{C_{z}}-\frac{R_{z} C_{z} z^{2}}{4 t^{2}}$ and $t$ is the time that takes for the signal to reach the dendrites of neuron B through the axon of neuron A. The potential will increase in the dendrites of neuron B by summing the potential until neuron B starts firing spikes to discharge the potential of its dendrites. Therefore the increase in the potential of the dendrites in neuron $\mathrm{B}$ due to the signal reached to neuron $\mathrm{B}$ through the axon of neuron A given in (B-2), and by using equation (B-4), can be approximated as:

$$
w_{o}(z, t)=v_{h}(z, t) \sum_{n=1}^{N} \exp \left(a T_{n}\right)+\frac{v_{h}(z, t)}{2 t} \sum_{n=1}^{N} T_{n} \exp \left(a T_{n}\right)
$$


By assuming that $a T_{n}<<1$ equation (B-5) can be approximated as:

$$
w_{o}(z, t) \approx v_{h}(z, t) \sum_{n=1}^{N}\left(1+a T_{n}\right)+\frac{v_{h}(z, t)}{2 t} \sum_{n=1}^{N} T_{n}\left(1+a T_{n}\right)=v_{h}(z, t) \sum_{n=1}^{N}\left(1+(a+1) T_{n}+a T_{n}^{2}\right)
$$

Or

$$
w_{o}(z, t)=v_{h}(z, t)\left(N+N(a+1) \bar{T}+N a\left(\sigma^{2}+\bar{T}\right)=N v_{h}(z, t)\left(1+(2 a+1) \bar{T}+a \sigma^{2}\right)\right.
$$

\section{Appendix C: Proof of theorem 3}

Proof: The transfer function of $n$ cascaded layers of neurons is written as:

$$
\begin{aligned}
L_{1: n}\left(\omega_{x}, \omega_{y}, \omega_{z}, t\right) & =\frac{t^{n-1} \prod_{l=1}^{n}\left(a_{l}\right)}{\Gamma(n)\left(R_{z} C_{z}\right)^{n}} \exp \left(-\frac{G_{z} t}{C_{z}}\right) \exp \left(-\frac{\omega_{z}^{2} t}{R_{z} C_{z}}\right)\left\{\left(\frac{1}{\omega_{x}^{2}+\omega_{y}^{2}+b_{1}}\right)\left(\frac{1}{\omega_{x}^{2}+\omega_{y}^{2}+b_{2}}\right) \cdots\right. \\
& \left.\ldots\left(\frac{1}{\omega_{x}^{2}+\omega_{y}^{2}+b_{n}}\right)\right\}
\end{aligned}
$$

Let us now assume that $b_{i}$ is the smallest value among $b_{l}$ for $\forall l \in \mathrm{N}-\{i\}$. Therefore any $b_{l}$ can be written as:

$$
b_{l}=b_{i}+\varepsilon_{l} \quad 0<\varepsilon_{l}<1
$$

where $\varepsilon_{l}$ is a real positive small number. Our second assumption that various neural layers have similar electrical properties means that $\varepsilon_{l}$ values are small in comparison with $b_{i}$. Transfer function $(\mathrm{C}-1)$ can then be rewritten as: 


$$
\begin{gathered}
L_{1: n}\left(\omega_{x}, \omega_{y}, \omega_{z}, t\right)=\frac{t^{n-1} \prod_{l=1}^{n}\left(a_{l}\right)}{\Gamma(n)\left(R_{z} C_{z}\right)^{n}} \exp \left(-\frac{G_{z} t}{C_{z}}\right) \exp \left(-\frac{\omega_{z}^{2} t}{R_{z} C_{z}}\right)\left\{\left(\frac{1}{\omega_{x}^{2}+\omega_{y}^{2}+b_{i}}\right)\left(\frac{1}{\omega_{x}^{2}+\omega_{y}^{2}+b_{i}+\varepsilon_{1}}\right) \ldots\right. \\
\left.\ldots\left(\frac{1}{\omega_{x}^{2}+\omega_{y}^{2}+b_{i}+\varepsilon_{n}}\right)\right\}
\end{gathered}
$$

Or

$$
\begin{aligned}
L_{1: n}\left(\omega_{x}, \omega_{y}, \omega_{z}, t\right) & =t^{n-1} \exp \left(-\frac{G_{z} t}{C_{z}}\right) \exp \left(-\frac{\omega_{z}^{2} t}{R_{z} C_{z}}\right) \\
& \left\{\left(\frac{\prod_{l=1}^{n}\left(a_{l}\right) / \Gamma(n)\left(R_{z} C_{z}\right)^{n}}{\left(\omega_{x}^{2}+\omega_{y}^{2}+b_{i}\right)^{n}+f_{1}\left(\varepsilon_{1}, \varepsilon_{2}, \ldots, \varepsilon_{n}\right)\left(\omega_{x}^{2}+\omega_{y}^{2}+b_{i}\right)^{n-1}+\ldots+f_{n+1}\left(\varepsilon_{1}, \varepsilon_{2}, \ldots, \varepsilon_{n}\right)}\right)\right\}
\end{aligned}
$$

where $f_{1}, f_{2}, \ldots f_{n+1}$ are polynomial functions of $\varepsilon_{1}, \varepsilon_{2}, \ldots, \varepsilon_{n}$ so that

$$
\begin{aligned}
& f_{1}\left(\varepsilon_{1}, \varepsilon_{2}, \ldots, \varepsilon_{n}\right) \propto \varepsilon_{1}+\varepsilon_{2}+\ldots+\varepsilon_{n} \\
& f_{2}\left(\varepsilon_{1}, \varepsilon_{2}, \ldots, \varepsilon_{n}\right) \propto \varepsilon_{1} \varepsilon_{2}+\varepsilon_{2} \varepsilon_{3}+\ldots+\varepsilon_{n-1} \varepsilon_{n} \\
& f_{3}\left(\varepsilon_{1}, \varepsilon_{2}, \ldots, \varepsilon_{n}\right) \propto \varepsilon_{1} \varepsilon_{2} \varepsilon_{3}+\varepsilon_{2} \varepsilon_{3} \varepsilon_{4}+\ldots+\varepsilon_{n-2} \varepsilon_{n-1} \varepsilon_{n} \\
& \ldots \\
& f_{n+1}\left(\varepsilon_{1}, \varepsilon_{2}, \ldots, \varepsilon_{n}\right) \propto \varepsilon_{1} \varepsilon_{2} \varepsilon_{3} \ldots \varepsilon_{n-2} \varepsilon_{n-1} \varepsilon_{n}
\end{aligned}
$$

Due to small values associated with $\varepsilon_{l}$, only the first few $f_{n} \mathrm{~s}$ in the denominator of $(\mathrm{C}-2)$ (with low $n$ values) have more significant values. The remaining of $f_{n}$ s becomes negligible as $n$ increases. Therefore transfer function (C-2) is approximated as: 


$$
\begin{aligned}
L_{1: n}\left(\omega_{x}, \omega_{y}, \omega_{z}, t\right) & \approx t^{n-1} \exp \left(-\frac{G_{z} t}{C_{z}}\right) \exp \left(-\frac{\omega_{z}^{2} t}{R_{z} C_{z}}\right) \\
& \left\{\left(\frac{\prod_{l=1}^{n}\left(a_{l}\right)}{\left(\omega_{x}^{2}+\omega_{y}^{2}+b_{i}\right)^{n}+\sum_{j=1}^{P} f_{j}\left(\varepsilon_{1}, \varepsilon_{2}, \ldots, \varepsilon_{n}\right)\left(\omega_{x}^{2}+\omega_{y}^{2}+b_{i}\right)^{n-j}}\right)\right\}
\end{aligned}
$$

where $P<<n$. By approaching $n$ to infinity, it is straightforward to see $\left(\omega_{x}^{2}+\omega_{y}^{2}+b_{i}\right)^{n-j} \approx\left(\omega_{x}^{2}+\omega_{y}^{2}+b_{i}\right)^{n}$. Therefore transfer function (C-3) is approximated as:

$$
\begin{aligned}
L_{1: n}\left(\omega_{x}, \omega_{y}, \omega_{z}, t\right) & \approx t^{n-1} \exp \left(-\frac{G_{z} t}{C_{z}}\right) \exp \left(-\frac{\omega_{z}^{2} t}{R_{z} C_{z}}\right)\left\{\left(\frac{\prod_{l=1}^{n}\left(a_{l}\right) / \Gamma(n)\left(R_{z} C_{z}\right)^{n}}{\left(\omega_{x}^{2}+\omega_{y}^{2}+b_{i}\right)^{n}\left(1+\sum_{k=1}^{P} f_{k}\left(\varepsilon_{1}, \varepsilon_{2}, \ldots, \varepsilon_{n}\right)\right)}\right)\right\} \\
& =\left\{\left(\frac{\left.\prod_{l=1}^{n}\left(a_{l}\right) / \Gamma(n)\left(b_{i} R_{z} C_{z}\right)^{n}\right)}{\left(\left(\frac{\omega_{x}}{b_{i}}\right)^{2}+\left(\frac{\omega_{y}}{b_{i}}\right)^{2}+1\right)^{n}\left(1+\sum_{k=1}^{P} f_{k}\left(\varepsilon_{1}, \varepsilon_{2}, \ldots, \varepsilon_{n}\right)\right)}\right)\right\}
\end{aligned}
$$

The term $\left(\left(\frac{\omega_{x}}{b_{i}}\right)^{2}+\left(\frac{\omega_{y}}{b_{i}}\right)^{2}+1\right)^{n}$ in the denominator in equation (C-4) can be expanded by using binomial series:

$$
L_{1: n}\left(\omega_{x}, \omega_{y}, \omega_{z}, t\right)=\frac{t^{n-1} \exp \left(-\frac{G_{z} t}{C_{z}}\right) \exp \left(-\frac{\omega_{z}^{2} t}{R_{z} C_{z}}\right)\left(\prod_{l=1}^{n}\left(a_{l}\right) / \Gamma(n)\left(b_{i} R_{z} C_{z}\right)^{n}\right)}{\left(1+\sum_{k=1}^{P} f_{k}\left(\varepsilon_{1}, \varepsilon_{2}, \ldots, \varepsilon_{n}\right)\right) \sum_{k=0}^{n}\left(\begin{array}{l}
n \\
k
\end{array}\right)\left(\left(\frac{\omega_{x}}{b_{i}}\right)^{2}+\left(\frac{\omega_{y}}{b_{i}}\right)^{2}\right)^{k}}
$$

where $\left(\begin{array}{l}n \\ k\end{array}\right)=\frac{n !}{k !(n-k) !}$. 
Let us set $X=n\left(\left(\frac{\omega_{x}}{b_{i}}\right)^{2}+\left(\frac{\omega_{y}}{b_{i}}\right)^{2}\right)$. Therefore equation (C-5) is rewritten as:

$$
\begin{aligned}
L_{1: n}\left(\omega_{x}, \omega_{y}, \omega_{z}, t\right) & =\frac{t^{n-1} \exp \left(-\frac{G_{z} t}{C_{z}}\right) \exp \left(-\frac{\omega_{z}^{2} t}{R_{z} C_{z}}\right)\left(\prod_{l=1}^{n}\left(a_{l}\right) / \Gamma_{\Gamma(n)\left(b_{i} R_{z} C_{z}\right)^{n}}\right)}{\left(1+\sum_{k=1}^{P} f_{k}\left(\varepsilon_{1}, \varepsilon_{2}, \ldots, \varepsilon_{n}\right)\right) \sum_{k=0}^{n}\left(\begin{array}{l}
n \\
k
\end{array}\right) \frac{X^{k}}{n^{k}}} \\
& =\frac{t^{n-1} \exp \left(-\frac{G_{z} t}{C_{z}}\right) \exp \left(-\frac{\omega_{z}^{2} t}{R_{z} C_{z}}\right)\left(\prod_{l=1}^{n}\left(a_{l}\right) / \Gamma(n)\left(b_{i} R_{z} C_{z}\right)^{n}\right.}{\left(1+\sum_{k=1}^{P} f_{k}\left(\varepsilon_{1}, \varepsilon_{2}, \ldots, \varepsilon_{n}\right)\right) \sum_{k=0}^{n} \frac{n !}{k !(n-k) !} \frac{X^{k}}{n^{k}}}
\end{aligned}
$$

By approaching $n$ to infinity, $L_{1: n}\left(\omega_{x}, \omega_{y}, \omega_{z}, t\right)$ in $(\mathrm{C}-6)$ approaches to the following function:

$$
\begin{aligned}
\lim L_{1: n}\left(\omega_{x}, \omega_{y}, \omega_{z}, t\right) & =\frac{t^{n-1} \exp \left(-\frac{G_{z} t}{C_{z}}\right) \exp \left(-\frac{\omega_{z}^{2} t}{R_{z} C_{z}}\right)\left(\prod_{l=1}^{n}\left(a_{l}\right) / \Gamma_{(n)\left(b_{i} R_{z} C_{z}\right)^{n}}\right)}{\left(1+\sum_{k=1}^{P} f_{k}\left(\varepsilon_{1}, \varepsilon_{2}, \ldots, \varepsilon_{n}\right)\right) \lim \sum_{k=0}^{n} \frac{n !}{k !(n-k) !} \frac{X^{k}}{n^{k}}} \\
& =\frac{t^{n-1} \exp \left(-\frac{G_{z} t}{C_{z}}\right) \exp \left(-\frac{\omega_{z}^{2} t}{R_{z} C_{z}}\right)\left(\prod_{l=1}^{n}\left(a_{l}\right) / \Gamma(n)\left(b_{i} R_{z} C_{z}\right)^{n}\right)}{\left(1+\sum_{k=1}^{P} f_{k}\left(\varepsilon_{1}, \varepsilon_{2}, \ldots, \varepsilon_{n}\right)\right) \sum_{k=0}^{\infty} \frac{1}{k !} \lim _{n \rightarrow \infty} \frac{n(n-1) \ldots(n-k+1)}{n^{k}} X^{k}} \\
& =\frac{t^{n-1} \exp \left(-\frac{G_{z} t}{C_{z}}\right) \exp \left(-\frac{\omega_{z}^{2} t}{R_{z} C_{z}}\right)\left(\prod_{l=1}^{n}\left(a_{l}\right) / \Gamma(n)\left(b_{i} R_{z} C_{z}\right)^{n}\right)}{\left(1+\sum_{k=1}^{P} f_{k}\left(\varepsilon_{1}, \varepsilon_{2}, \ldots, \varepsilon_{n}\right)\right) \sum_{k=0}^{\infty} \frac{X^{k}}{k !}}
\end{aligned}
$$

The denominator in equation (C-7) is the Taylor series for an exponential function, i.e., equation (C-7) can be rewritten as:

$$
\lim L_{1: n}\left(\omega_{\substack{x \\ n \rightarrow+\infty}}, \omega_{z}, t\right)=\frac{t^{n-1} \exp \left(-\frac{G_{z} t}{C_{z}}\right) \exp \left(-\frac{\omega_{z}^{2} t}{R_{z} C_{z}}\right)\left(\prod_{l=1}^{n}\left(a_{l}\right) / \Gamma(n)\left(b_{i} R_{z} C_{z}\right)^{n}\right) \exp (-X)}{\left(1+\sum_{k=1}^{P} f_{k}\left(\varepsilon_{1}, \varepsilon_{2}, \ldots, \varepsilon_{n}\right)\right)}
$$


By replacing $X$ with $n\left(\left(\frac{\omega_{x}}{b_{i}}\right)^{2}+\left(\frac{\omega_{y}}{b_{i}}\right)^{2}\right)$, we arrive at the following equation:

$$
\begin{aligned}
& \lim L_{1: n}\left(\omega_{x}, \omega_{y}, \omega_{z}, t\right)=\lim \left\{\frac{t^{n-1}\left(\prod_{l=1}^{n}\left(a_{l}\right) / \Gamma(n)\left(b_{i} R_{z} C_{z}\right)^{n}\right)}{\left(1+\sum_{k=1}^{P} f_{k}\left(\varepsilon_{1}, \varepsilon_{2}, \ldots, \varepsilon_{n}\right)\right)} \exp \left(-\frac{G_{z} t}{C_{z}}\right)\right. \\
& \left.\times \exp \left(-n\left(\left(\frac{\omega_{x}}{b_{i}}\right)^{2}+\left(\frac{\omega_{y}}{b_{i}}\right)^{2}+\left(\frac{\omega_{z}}{b_{z}}\right)^{2}\right)\right)\right\}
\end{aligned}
$$

where $b_{z}=\sqrt{\frac{R_{z} C_{z}}{n t}}$.

Repeated convolutions exploited in this theorem, can also be found in the central limit theorem (CLT) (Papoulis 1962). However regarding the CLT and this theorem, there are some issues we need to notice:

1) CLT is established in a statistical setting; however theorem 3 is proved in a completely deterministic framework.

2) CLT is accomplished by adding a large number of iid random variables; however theorem 3 is concluded as a result of the KVL and KCL equations derived from consecutive neural layers characterized with some linear electrical properties.

3) Probability distribution functions corresponding to the appropriate random variables are convolved together in CLT. However the electrical impulse response of each neural layer is convolved with the impulse responses of other neural layers in early visual system according to theorem 3 .

4) In CLT, the probability distribution functions can be a wide range of admissible functions. However due to the conditions imposed by the electrical properties (KCL 
and KVL equations) of neural layers, modified Bessel funtions are the only kernels acceptable in theorem 3.

\section{References}

Adelson E, Bergen J (1985) Spaio-temporal Energy Models for the Perception of Motion. Journal of Optical Society of America A, Vol. 2, pp. 284-299.

Baer SM, Rinzel J (1991) Propagation of Dendritic Spikes Mediated by Excitable Spines: A Continuum Theory. Journal of Neurophysiology, Vol. 65, No. 4, pp 874-890.

Caceci $\mathrm{T}$ (1998) Anatomy and Physiology of the Eye. (http://www.vetmed.vt.edu/education/Curriculum/VM8054/EYE/EYEDEMO.HTM),

Virginia/Marylan Regional College of Veterinary Medicine.

Carandini M, Ferster D (2000) Membrane Potential and Firing Rate in Cat Primary Visual Cortex. Journal of Neuroscience, Vol. 20, No. 1, pp. 470-484.

Carandini M, Heeger DJ (2012) Normalization as a Canonical Neural Computation. Nature Review Neuroscience, Vol. 13, pp. 51-62.

Conway BR, Livingstone MS (2006) Spatial and Temporal Properties of Cone Signals in Alert Macaque Primary Visual Cortex. Journal of Neuroscience, Vol. 25, No. 42, 10826-10846.

DeAngelis GC, Anzai A (2004) A Modern View of the Classical Receptive Field: Linear and nonLinear Spatio-temporal Processing by V1 Neurons. In L.M. Chalupa, and J.S. Werner (eds.), The Visual Neurosciences, Vol 1, MIT Press, Cambridge, pp. 704-719.

DeAngelis DC, Ohzawa I, Freeman RD (1995) Receptive-field Dynamics in the Central Visual Pathways. Trends in Neurosciences, Vol. 18, pp:451-457.

Dinse HRO,von Seelen W (1981) On the function of cell systems in area 18, parts I and II. Biological Cybernetic, Vol. 41, pp.47-69.

Duits R, Florack L, de Graaf J, ter Haar Romeny B (2004) On the Axioms of Scale Space Theory, Journal of Mathematical Imaging and Vision, Vol. 22, pp. 267-298. 
Enroth-Cugell C, Robson JG (1966) The Contrast Sensitivity of Retinal Ganglion Cells of the Cat. Journal of Physiology, Vol. 187, pp 517-552.

Florack L, ter Haar Romney B, Koenderink J, Viergever M (1992) Scale and the Differential Structure of Images, Image and Vision Computing, Vol. 10, No. 6, pp. 376-388.

Florack, L (1997) Image Structure. Series in Mathematical Imaging and Vision, Springer, Dordrecht.

Granit R, Kernell D, Shortess GK (1963) Quantitative Aspects of Repetitive Firing of Mammalian Motoneurons caused by Injected Currents. Journal of Physiology (London), Vol. 168, pp. 911931

Hartline HK (1938) The Response of Single Optic Nerve Fibres of the Vertebrate Eye to Illumination of the Retina. American Journal of Physiology, Vol. 121, pp. 400-415.

Hubel DH, Wiesel TN (2005) Brain and Visual Perception: the Story of a 25-year collaboration. Oxford University Press, Oxford.

Koenderink JJ (1988) Scale-Time, Biological Cybernetic, Vol. 58, pp. 159-162.

Koenderink JJ, Kappers A, van Doorn A (1992) Local Operators: The embodiment of Geometry. In: Orban GA, Nagel HH (ed) Artificial and Biological Vision Texte imprime,, Springer, pp. 1-23.

Krone G, Mallot HA, Palm G, Schutz A (1986) Spatio-temporal receptive fields: a dynamical model derived from cortical architectonics. Proceedings of Royal Society London Series B, Vol. 226, pp. 421-444.

Kuffler SW (1953) Discharge Patterns and Functional Organization of Mammalian Retina. Journal of Neurophysiology, Vol. 16, No. 1, pp. 37-68.

Lindeberg T, Fagerstrom D (1996) Scale-Space with Causal Time Direction, In: Proceedings of $4^{\text {th }}$ European Conference on Computer Vision, Vol. 1064, pp. 229-240, Springer, Berlin.

Lindeberg T (2011) Generalized Gaussian Scale-Space Axiomatic Comprising Linear Scale-Space, Affine Scale-Space and Spatio-Temporal Scale-Space. Journal of Mathematical Imaging and Vision, Vol. 40, pp: 36-81.

Lindeberg T (2013) A Computational Theory of Visual Receptive Fields. Biological Cybernetics, Vol. 107, pp: 589-635. 
Lindeberg T (2015) Separable Time-Causal and Time-Recursive Spatio-Temporal Receptive Fields, In Scale Space and Variational Methods in Computer Vision, Lecture Notes in Computer Science, Vol. 9087, pp. 90-102.

Mahmoodi S (2012) Edge Detection based on Mumford-Shah Green Function. SIAM Journal on Imaging Sciences, Vol. 5, No. 1, pp: 343-365.

Miller JP, Rall W, Rinzel J (1985) Synaptic Amplification by Active Membrane in Dendritic Spines. Brain Research, Vol. 325, pp. 325-330.

Mumford D, Shah J (1989) Optimal Approximations by Piecewise Smooth Functions and Associated Variational Problems. Communications on Pure and Applied Mathematics, Vol. 42, pp: 577-688.

Papoulis A (1962) The Fourier Integral and its Applications. McGraw-Hill Book Company, Inc..

Penrose R (2004) The Road to Reality: A Complete Guide to the Laws of Universe, Jonathan Cape London.

Segev I, Rall W (1988) Computational Study of an Excitable Dendritic Spine. Journal of Neurophysiology, Vol. 60, No. 2, pp: 499-523.

Sherrington CS (1906) The integrative action of the nervous system. C Scribner and Sons, New York.

Skilling HH (1979) Electric Transmission Lines: Distributed Constants, Theory and Applications. McGraw-Hill Book Company, Inc.

ter Haar Romeny B (2003) Front-End Vision and Multi-Scale Image Analysis. Springer Dordrecht.

ter Haar Romeny B, Florack L, Nielsen M (2001) Scale-time Kernels and Models, In: Scale-Space and Morphology, Proceedings of Scale-Space'01, Vancouver, Canada, LNCS, Springer, Berlin.

Nielsen M, Florack L, Deriche R, (1997) Regularization, Scale-Space and Edge Detection Filters, Journal of Mathematical Imaging and Vision, Vol. 7, pp. 291-307.

von Seelen W, Mallot HA, Giannakopoulos F. (1987) Characteristics of Neural Systems in the Visual Cortex. Biological Cybernetic, Vol. 56, pp. 37-49. 
Weickert J, Ishikawa S, Imiya A (1997), On the History Gaussian Scale-Space Aximatics, chapter 4, pp.45-59, in J. Sporring, M. Nielsen, L.M.J. Florack and P. Johansen (Eds.), Gaussian Scale Space Theory, Vol. 8, Computational Imaging and Vision Series, 1997, Kulwer.

Weickert J, Ishikawa S, Imiya A (1999) Linear Scale Space has First been proposed in Japan, Journal of Mathematical Imaging and Vision, Vol. 10, pp. 237-252.

Young RA (1987) The Gaussian Derivative Model for Spatial Vision: I. Retinal Mechanisms. Spatial Vision, Vol. 2, No. 4, pp:273-293.

Young RA, Lesperance RM, Meyer WW (2001) The Gaussian Derivative Model for SpatialTemporal Vision: I. Cortical Model. Spatial Vision, Vol. 14, No.3,4, pp:261-319. 

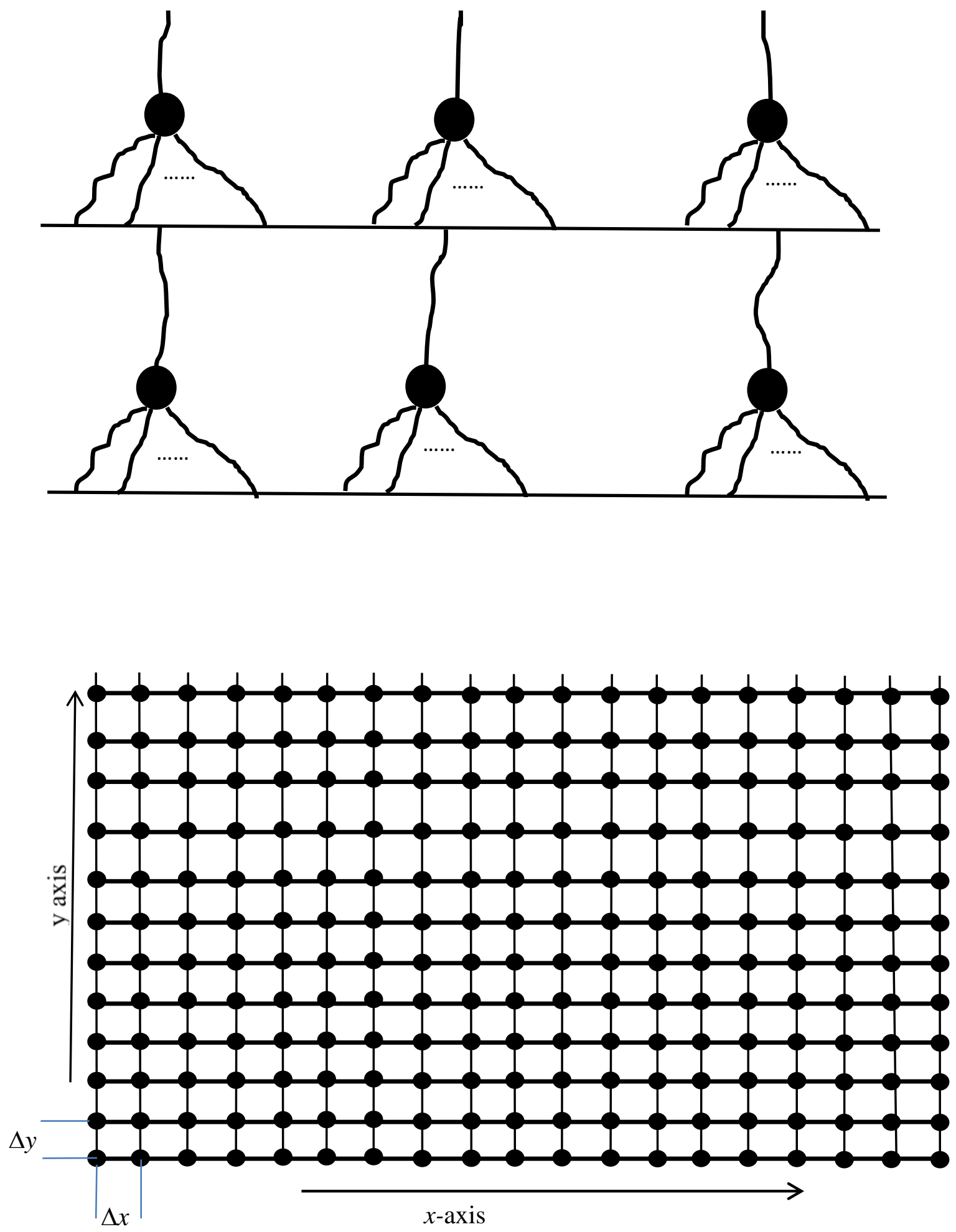

Fig (1): (top) Two dimensional block diagram of some small part of two typical layers of our model (bottom) A model of neural configuration for a single layer. The black spots represent the neurons which are connected to the neighbouring neurons through dendrites forming a two dimensional grid 


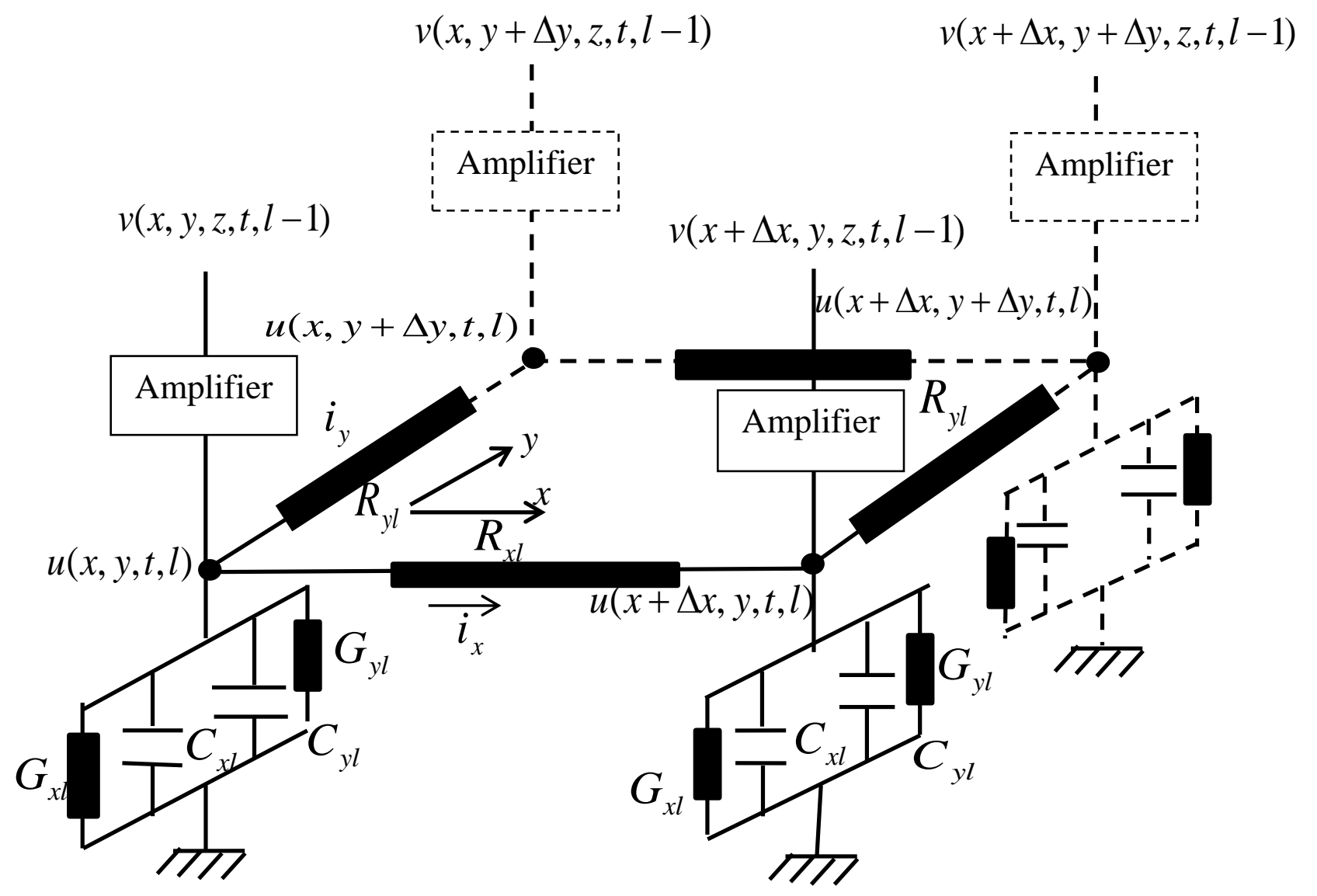

Fig (2): An electrical circuit modelling the retina by using a transmission (conductive) grid representing a neural layer

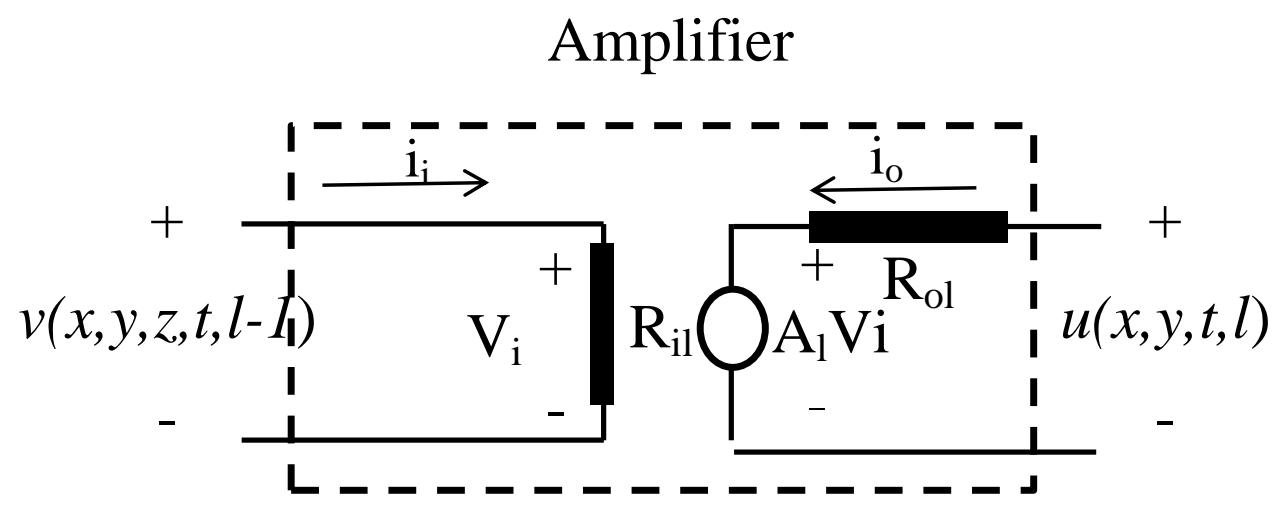

Fig (3): A model of a simple electrical amplifier for spine heads 

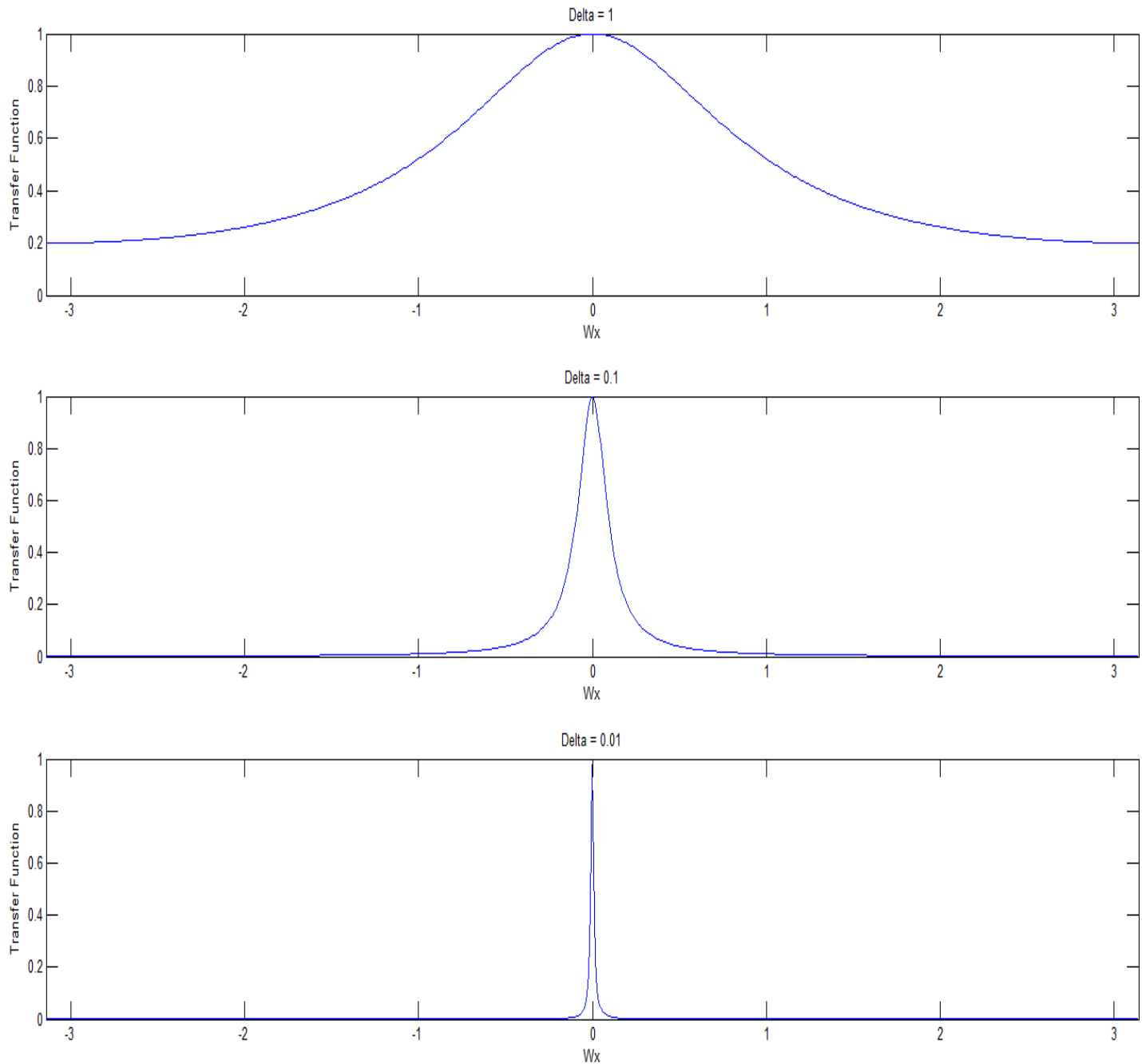

Fig (4): The transfer function $H$ calculated in equation (20) for $\omega_{y}=0$, $\frac{R_{l} A_{l}}{R_{o l}}=2 R_{l} G_{l}+\frac{R_{l}}{R_{o l}}=1$ and $\Delta=0.01,0.1$, and 1 . 


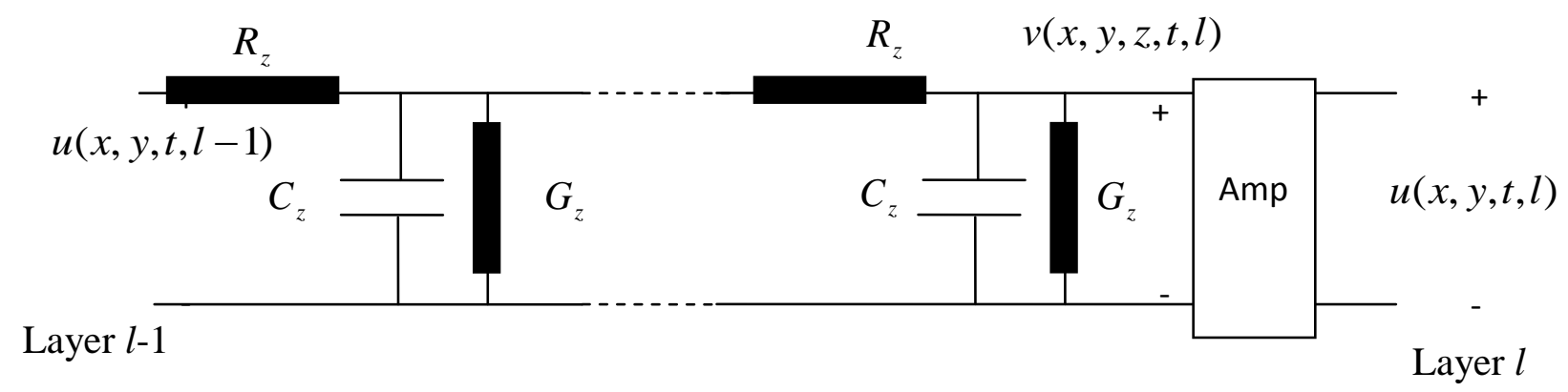

Fig (5): Axon modelled by a transmission line connecting two consecutive layers of dendrites

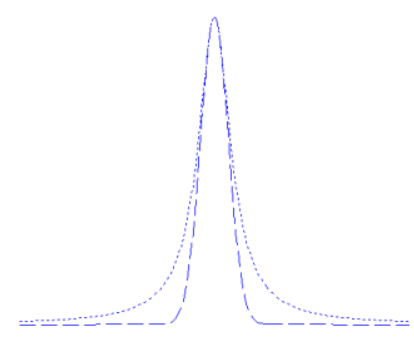

$a$

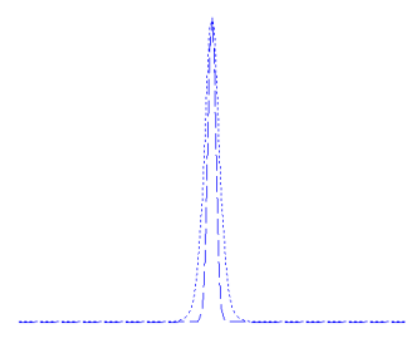

$d$

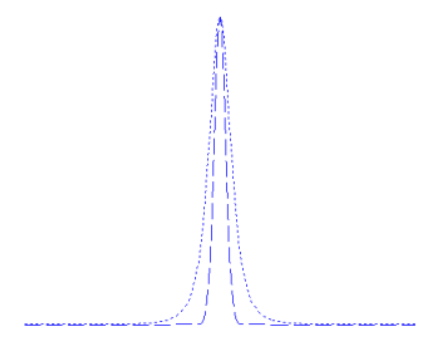

$b$

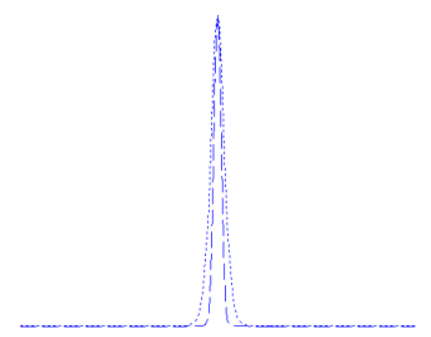

$e$

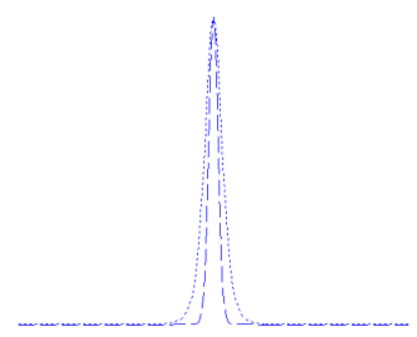

$c$

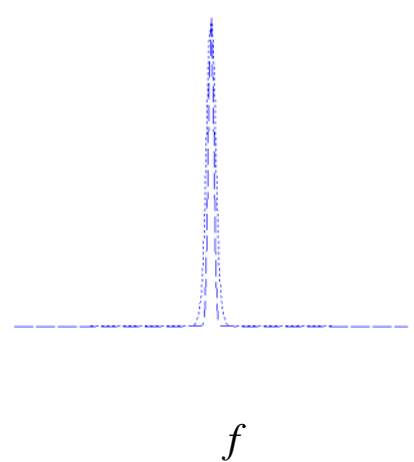

Fig (6): The filter obtained from the $l^{\text {th }}$ layer (dotted curve) is compared in frequency domain with a Gaussian filter (dashed curve) for the corresponding layer for a) $l=l$ b) $l=2$ c) $l=3$ d) $l=4$ e) $l=5$ f) $l=6$ 


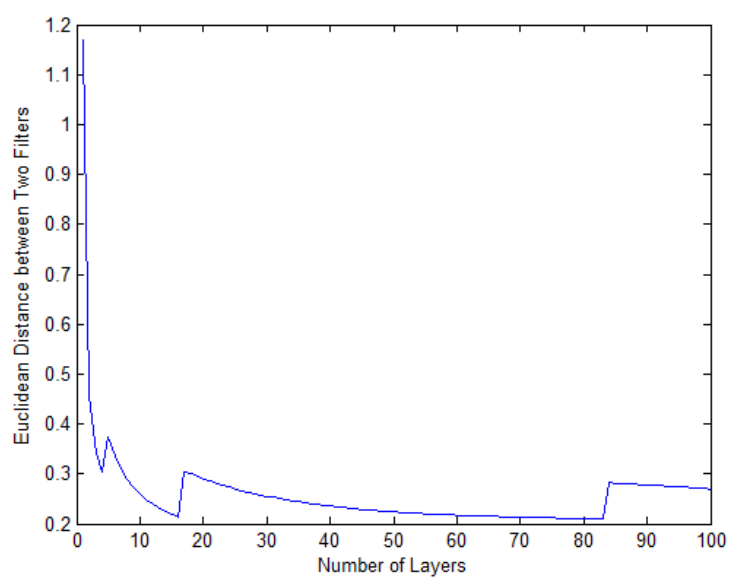

Fig (7): The Euclidean difference between the filter obtained from the $l^{\text {th }}$ layer and the Gaussian filter in frequency domain for various values of $l$

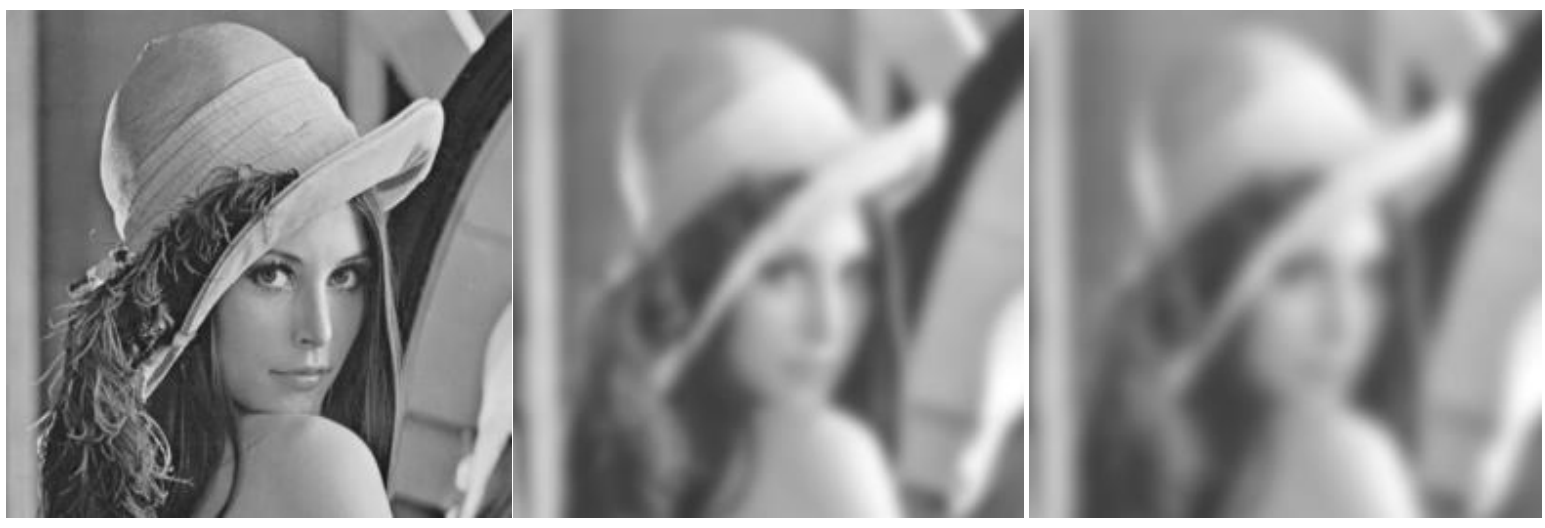

Fig (8): The filtered image in the $6^{\text {th }}$ layer (left) original image (middle) image filtered by equation (21) (right) image filtered by an equivalent Gaussian filter.

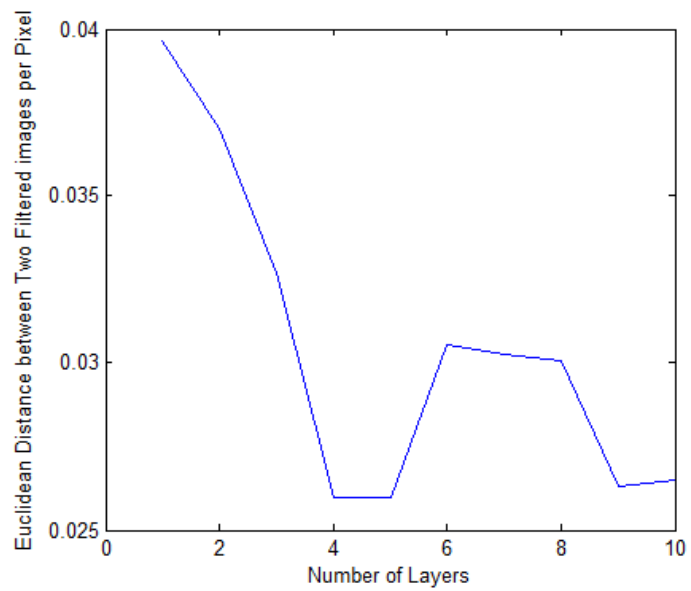

Fig (9): The difference between the two filtered images (by Gaussian filter and equation (10)) for the first ten layers 

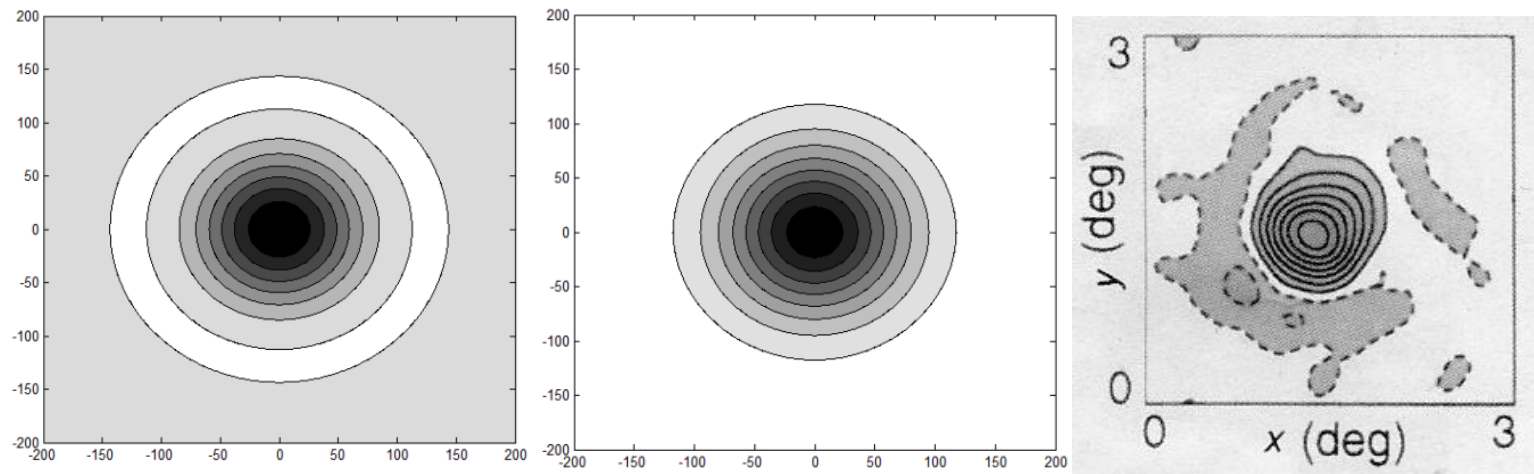

Fig (10): (Left) The Laplacian of a Gaussian over a spatial domain (middle) The Laplacian of $K_{n}(x, y)$ for $n=6$ (right) An example of a spatial receptive profile of an LGN cell as described in DeAngelis et al. (1995). 

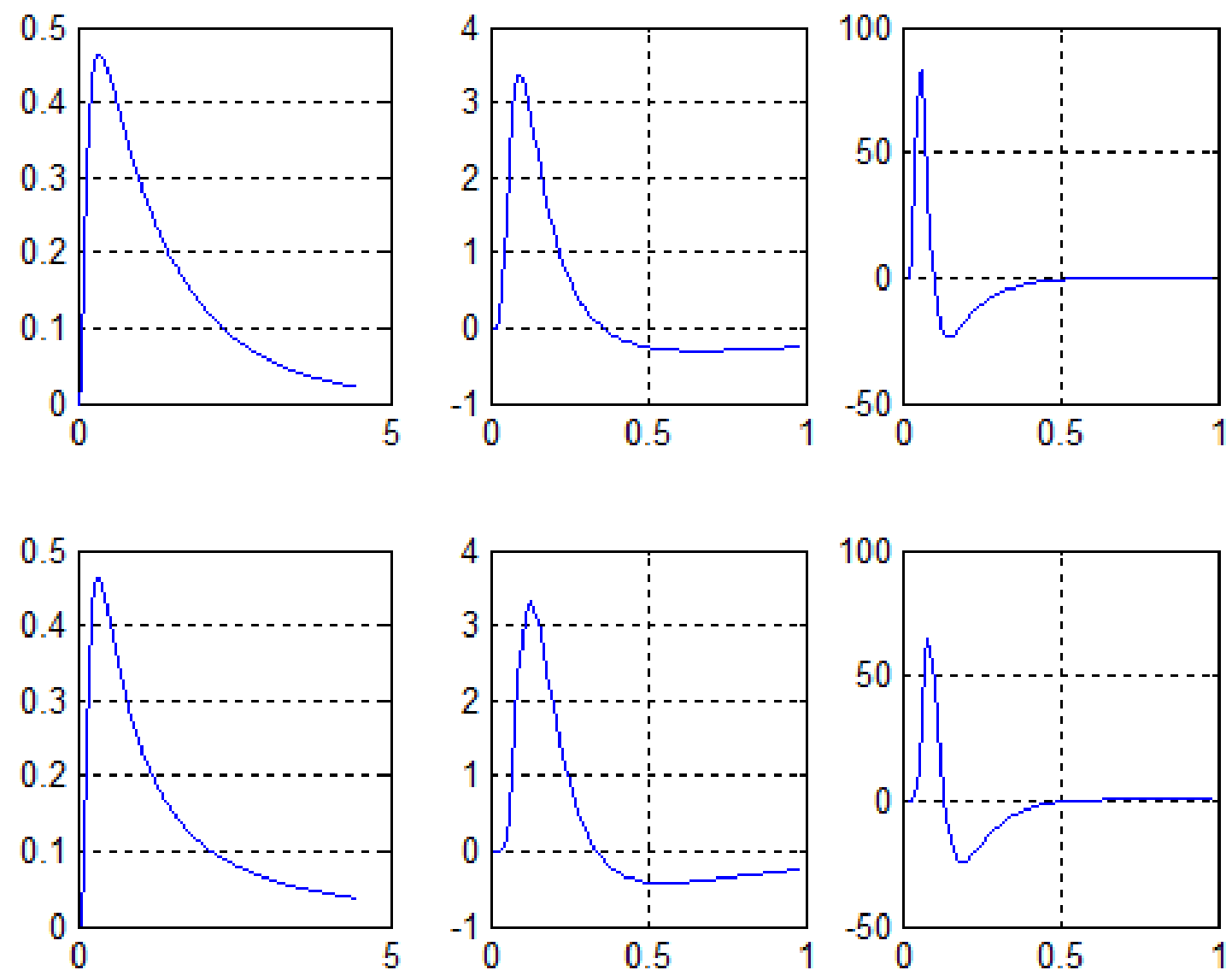

Fig (11): (top row: from left to right) Time causal smoothing kernel $v(z, t)=\frac{1}{2} \sqrt{\frac{1}{R_{z} C_{z} \pi t}} \exp \left(-\frac{G_{z} t}{C_{z}}\right) \exp \left(-\frac{R_{z} C_{z} z^{2}}{4 t}\right)$ with $R_{z} C_{z}=\frac{1}{6}, z=\sqrt{6}$, and $\frac{G_{z}}{C_{z}}=0.6$ and its first and second temporal derivatives (Bottom row: from left to right), time causal smoothing kernel $\varphi(t ; \tau)=\frac{\tau}{\sqrt{2 \pi t^{3}}} \exp \left(-\frac{\tau^{2}}{2 t}\right)$ with $\tau=1$ and its first and second temporal derivatives (Lindeberg 2011) 

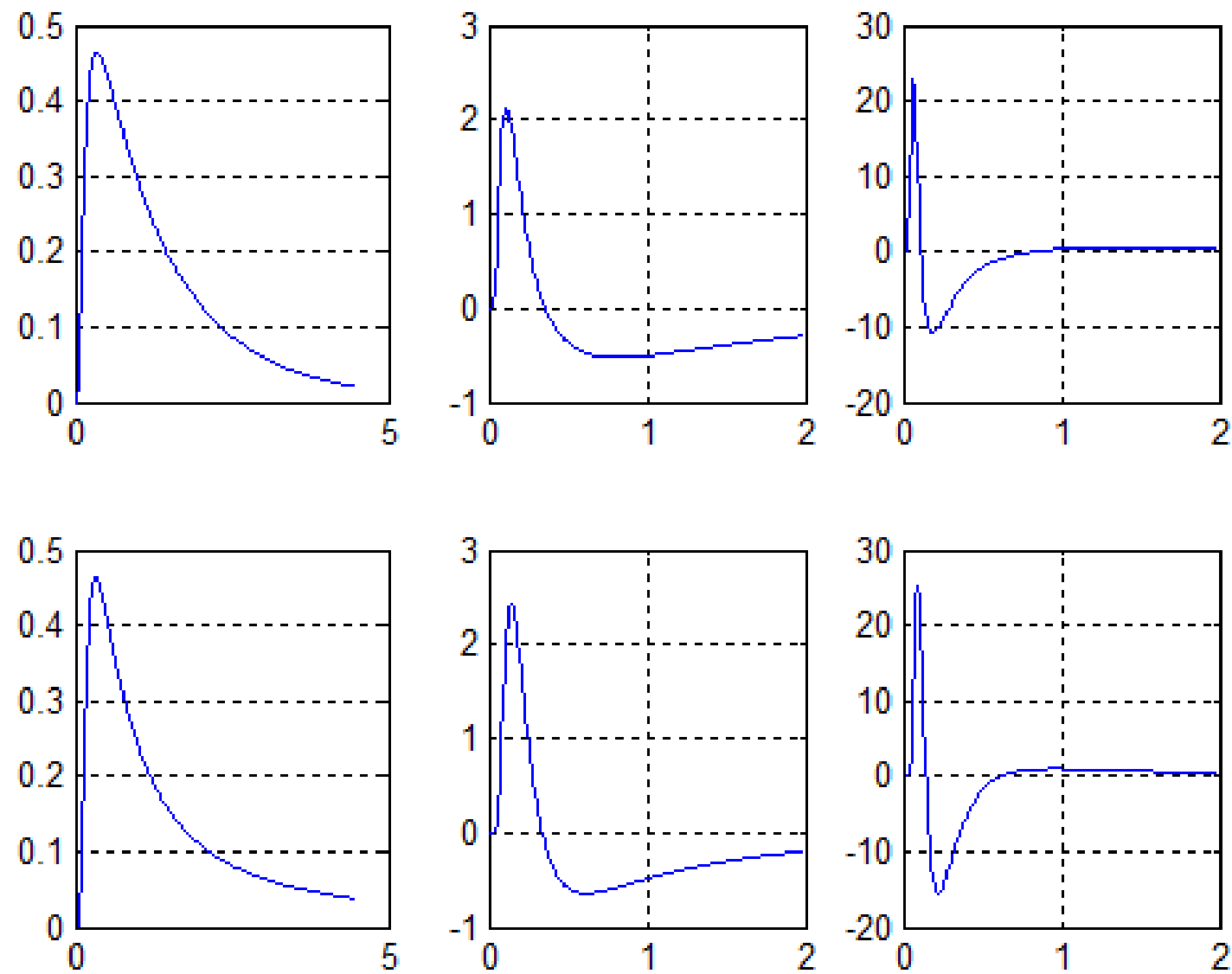

Fig (12): (Top row: from left to right) Time casual kernel $v(z, t)=\frac{1}{2} \sqrt{\frac{1}{\pi t R_{z} C_{z}}} \exp \left(-\frac{G_{z} t}{C_{z}}\right) \exp \left(-\frac{R_{z} C_{z} z^{2}}{4 t}\right)$ considered in figure (11) with the first and second derivatives with respect to $t^{\prime}=\sqrt{t}$ (Bottom row: from left to right), time casual kernel $\varphi(t ; \tau)=\frac{\tau}{\sqrt{2 \pi t^{3}}} \exp \left(-\frac{\tau^{2}}{2 t}\right)$ (Lindeberg 2011) considered in figure (11) with the first and second derivatives with respect to $t^{\prime}=\sqrt{t}$ 

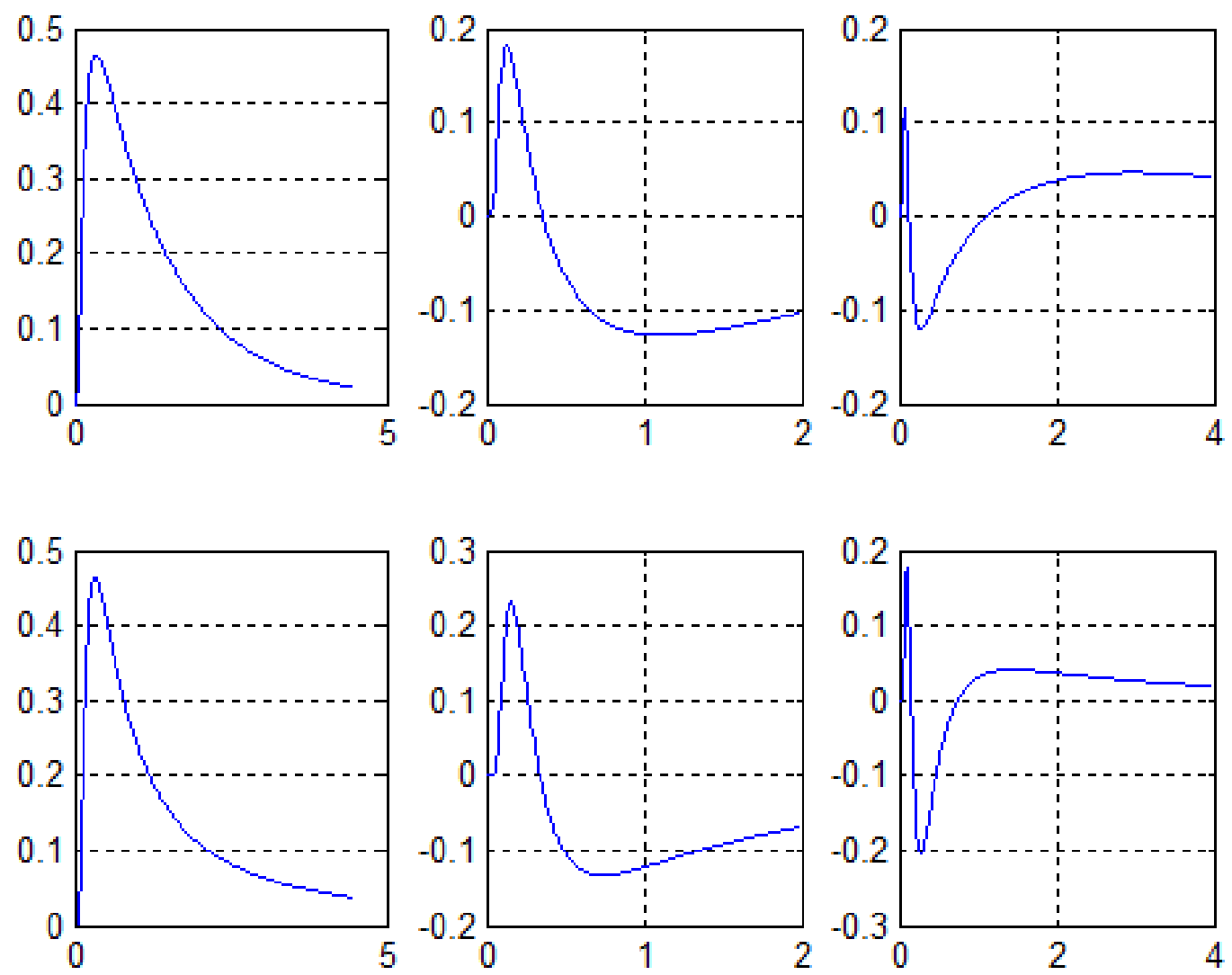

Fig (13): (Top row: from left to right) Time casual kernel $v(z, t)=\frac{1}{2} \sqrt{\frac{1}{\pi t R_{z} C_{z}}} \exp \left(-\frac{G_{z} t}{C_{z}}\right) \exp \left(-\frac{R_{z} C_{z} z^{2}}{4 t}\right)$ considered in figure (11) with the first and second derivatives with respect to $t^{\prime}=\log \left(\frac{t}{2}\right)$ (Bottom row: from left to right) time casual kernel $\varphi(t ; \tau)=\frac{\tau}{\sqrt{2 \pi t^{3}}} \exp \left(-\frac{\tau^{2}}{2 t}\right)$ (Lindeberg 2011) considered in figure (11) with the first and second derivatives with respect to $t^{\prime}=\log \left(\frac{t}{2}\right)$ 


\section{LGN}
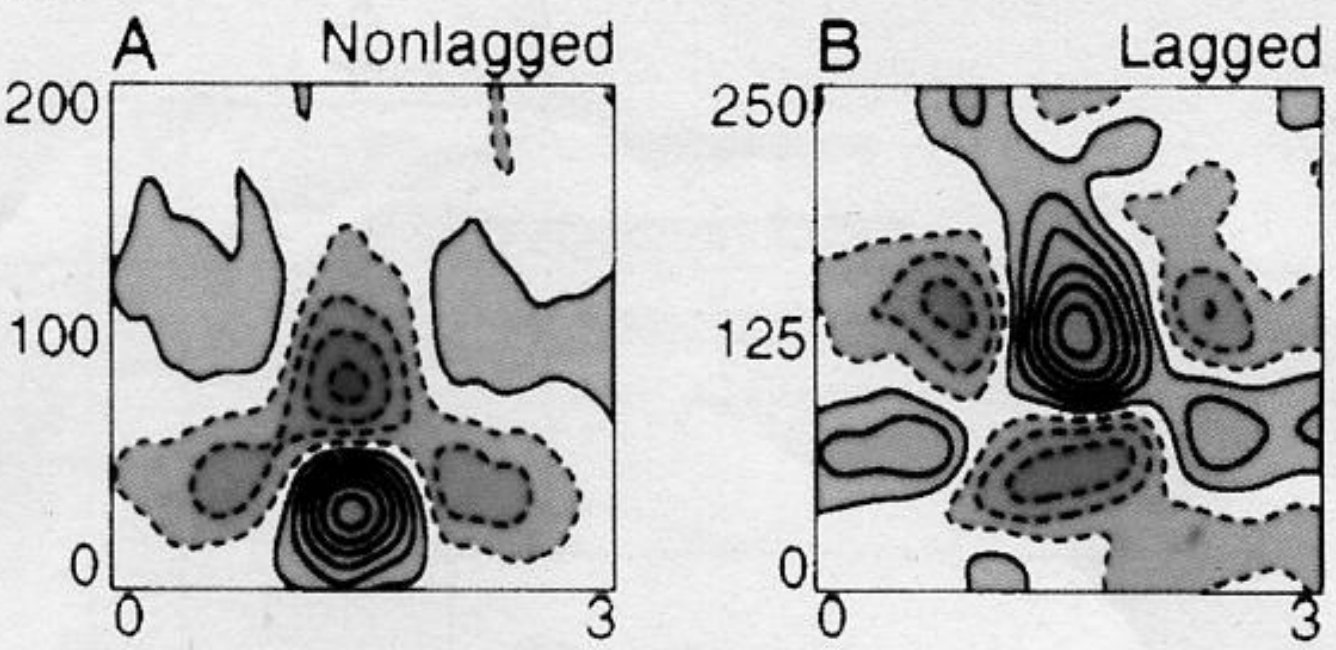

Fig (14): Examples of spatiotemporal receptive field profiles of nonlagged (left) and a lagged (right) LGN cells as stated by DeAngelis et al. (1995). 


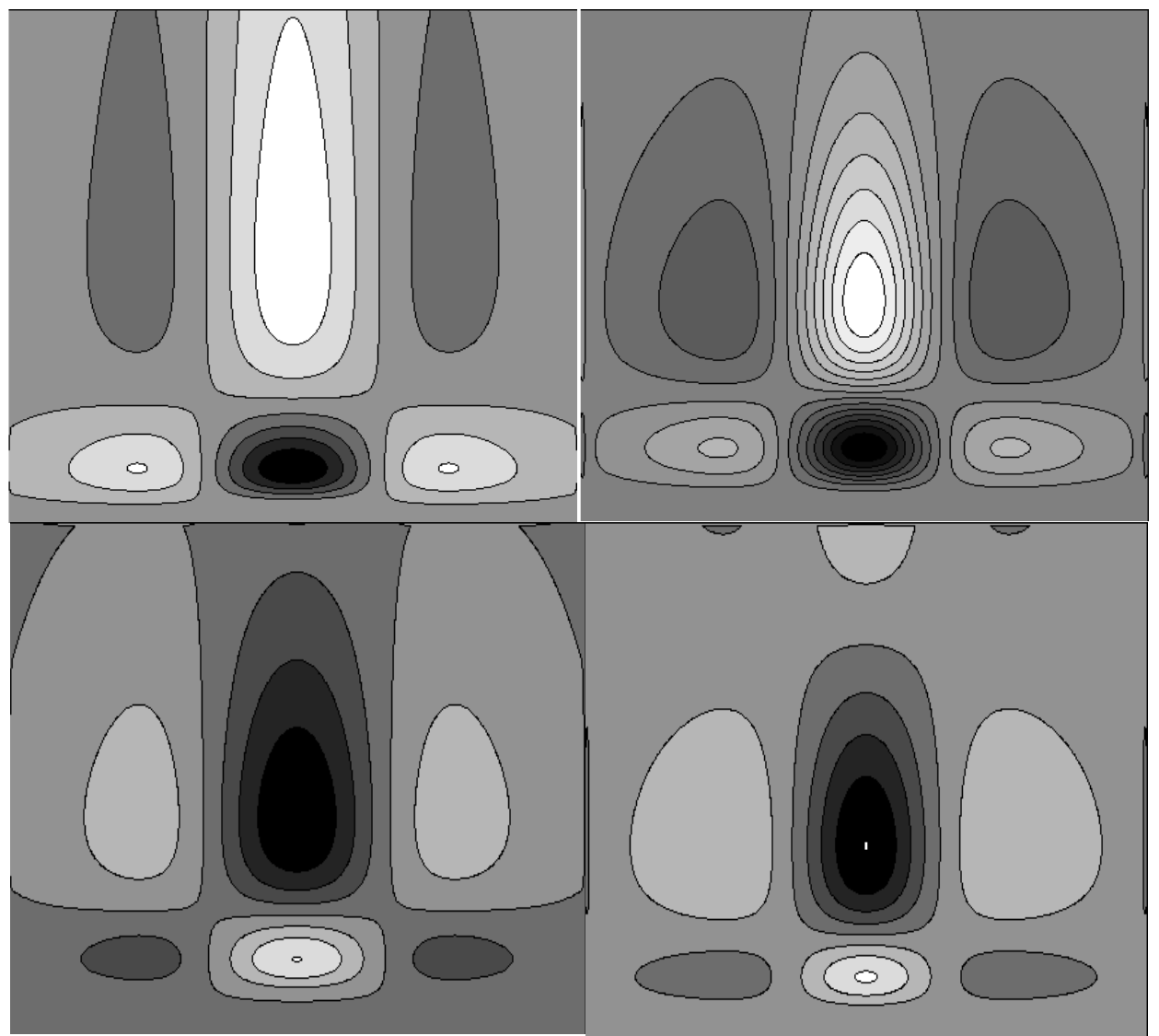

Fig (15): Space-time separable kernels (upper left) Time-causal spatio-temproal kernel $h_{x x t^{\prime}}(x, t ; s, \tau)=g_{x x}(x ; s) \phi_{t^{\prime}}(t ; \tau, \delta)$ with $s=0.3$ and $\tau=17$ (lower left) Time-causal spatiotemporal kernel $h_{x x t^{\prime} t^{\prime}}=g_{x x}(x ; s) \phi_{t^{\prime} t^{\prime}}(t ; \tau, \delta$ ) with $s=0.3$ and $\tau=25$, (upper right) Timecausal spatio-temporal kernel $h_{x x t^{\prime}}(x, t ; s, \tau)=\left(K_{n}\right)_{x x}(x ; s)\left(\psi_{n}\right)_{t^{\prime}}(t ; \tau, \delta)$ with $R_{z} C_{z} z^{2}=1200$, $\frac{G_{z}}{C_{z}}=0.015, \quad b_{i}=0.4$ and $n=6$ (lower right) Time causal spatio-temporal kernel $h_{x x t^{\prime} t^{\prime}}(x, t ; s, \tau)=\left(K_{n}\right)_{x x}(x ; s)\left(\psi_{n}\right)_{t^{\prime} t^{\prime}}(t ; \tau, \delta)$ with $R_{z} C_{z} z^{2}=1200, \frac{G_{z}}{C_{z}}=0.005, b_{i}=0.4$ and $n=6$ For the time-causal kernels, the temporal derivatives have been computed based on the transformed temporal derivative operator $\partial_{t^{\prime}} \sim t^{k} \partial_{t}$ here with $k=1 / 2$. 

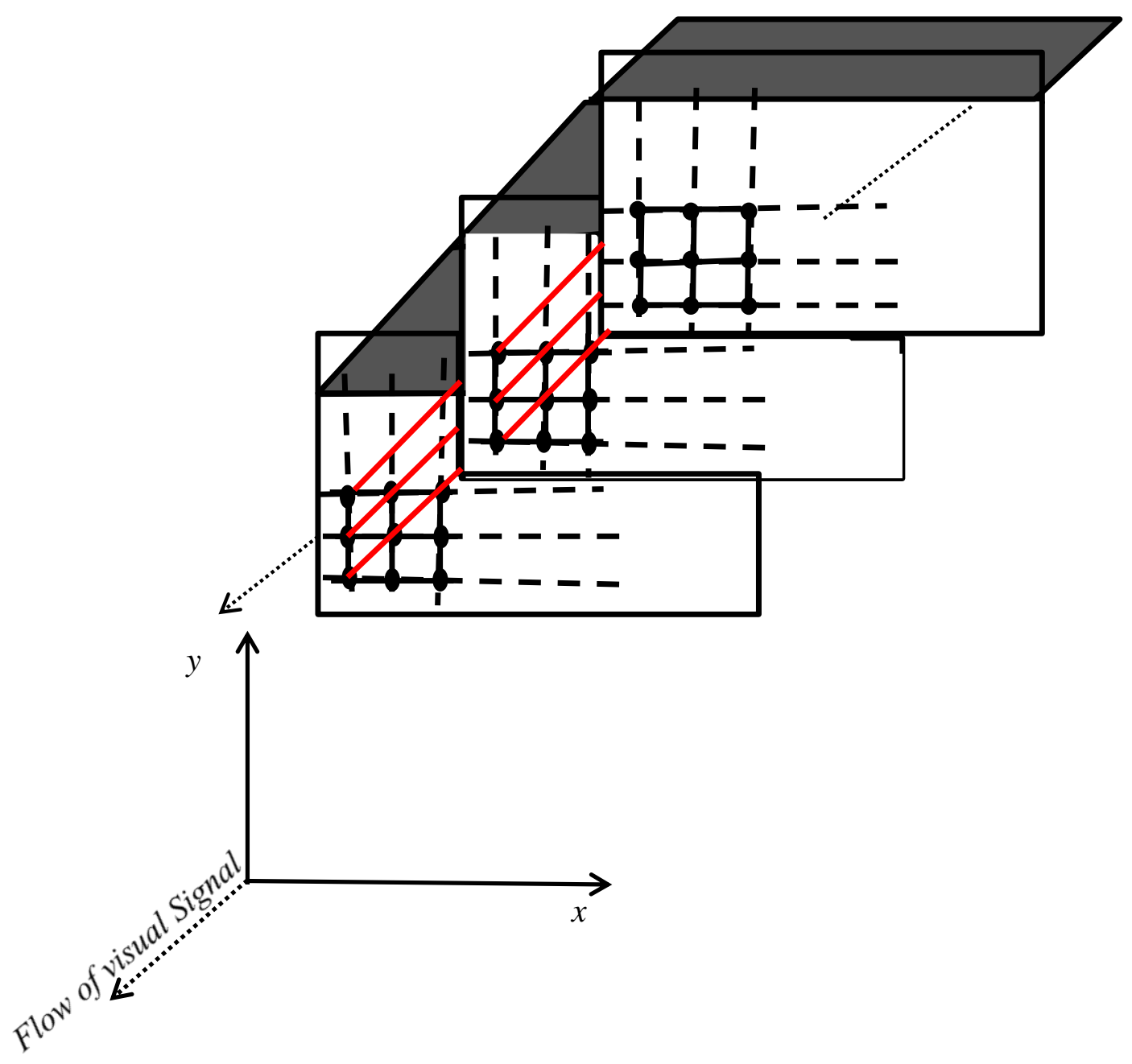

Fig (16): (top) Three consecutive layers of the neural network configuration in our model, the black circles, black lines and red lines represent neurons, dendrites and axons respectively (middle) A model for a non-isotropic neuron in space corresponding to one of the vertical planes in figure (16-top) (bottom) A model for motion selective neurons corresponding to the gray horizontal plane in figure (16-top) 

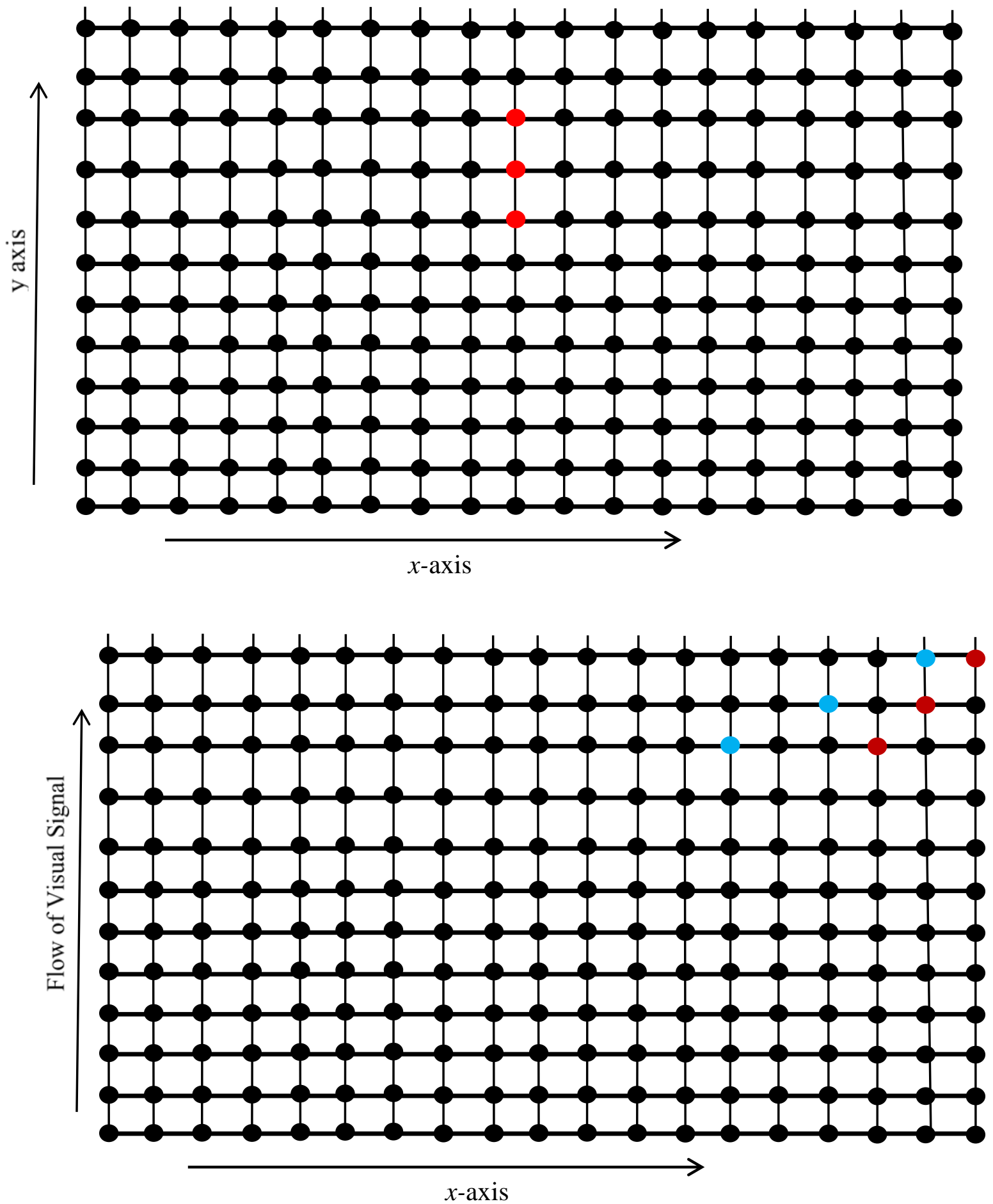

Fig (16) (continued): (top) Three consecutive layers of the neural network configuration in our model, the black circles, black lines and red lines represent neurons, dendrites and axons respectively (middle) A model for a non-isotropic neuron in space corresponding to one of the vertical planes in figure (16-top) (bottom) A model for motion selective neurons corresponding to the gray horizontal plane in figure (16-top) 


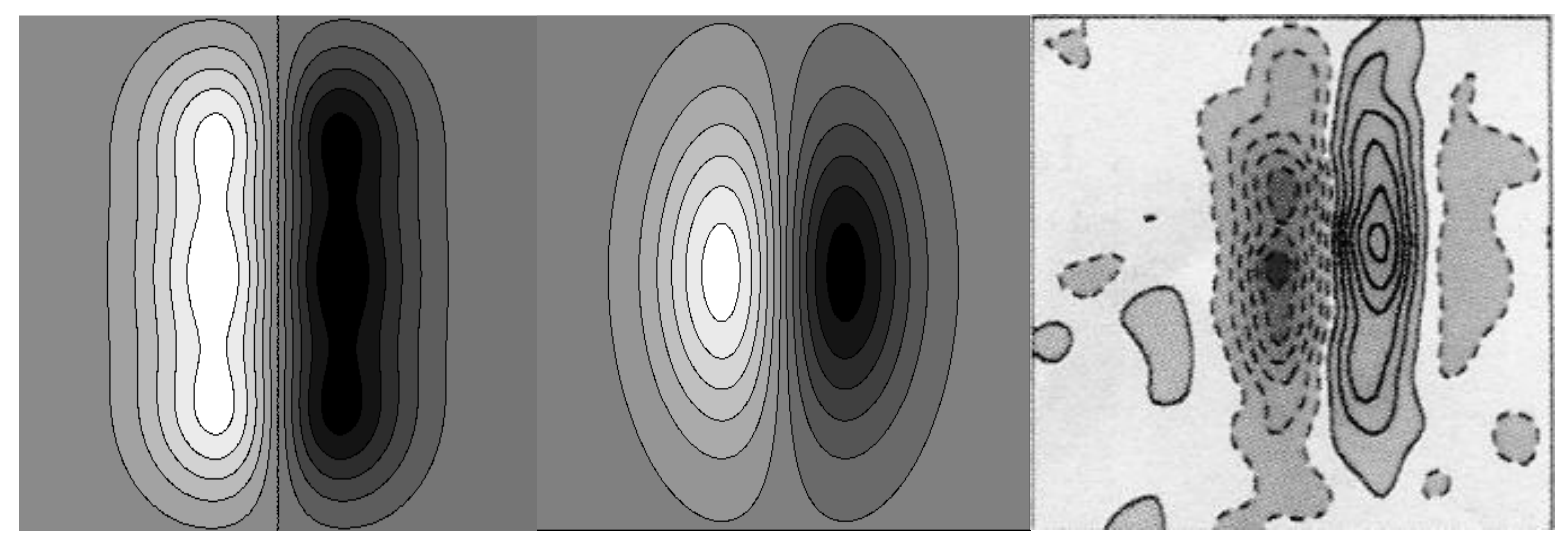

Fig (17): First order directional derivative along $x$ direction (left) of a non-isotropic elongated filter combined by three isotropic neurons for $\mu=130, n=10$, and $d=10$, (middle) of a nonisotropic elongated Gaussian with $\frac{\sigma_{y}}{\sigma_{x}}=3$, (right) an example of the receptive field profile of a simple cell with strong directional preference as reported in (DeAngelis et al. 1995) 


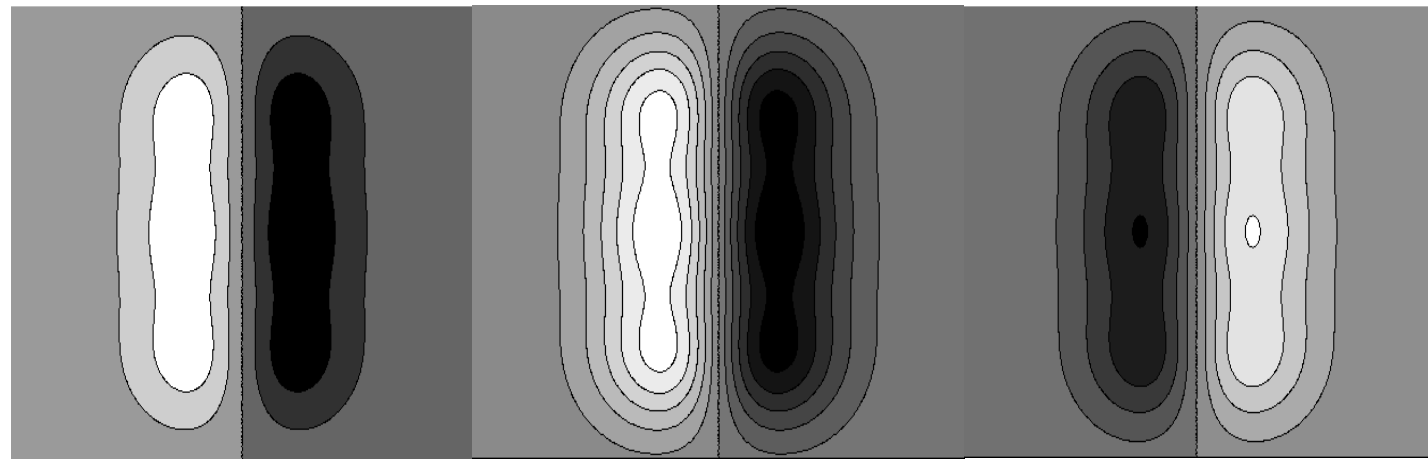

$\mathrm{T}=36 \mathrm{~ms} \quad \mathrm{~T}=110 \mathrm{~ms} \quad \mathrm{~T}=210 \mathrm{~ms}$

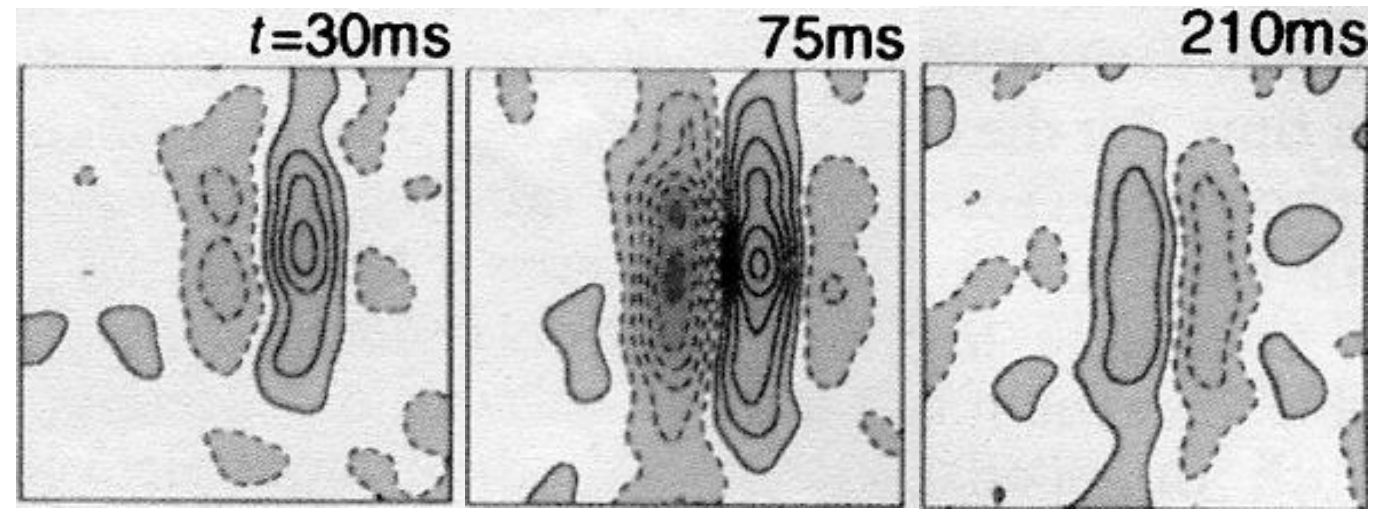

Fig (18): (top) Spatio-temporal response of a non-isotopic separable elongated neuron shown in figure (16-top) for $y_{d}=10, n=10, R_{z} C_{z}=1.9, \frac{G_{z}}{C_{z}}=0.001, R_{z} C_{z} z^{2}=400$ and $\mu=130$ (bottom) Examples of spatiotemporal receptive profile of a separable non-isotropic neuron as reported by DeAngelis et al. (1995). 

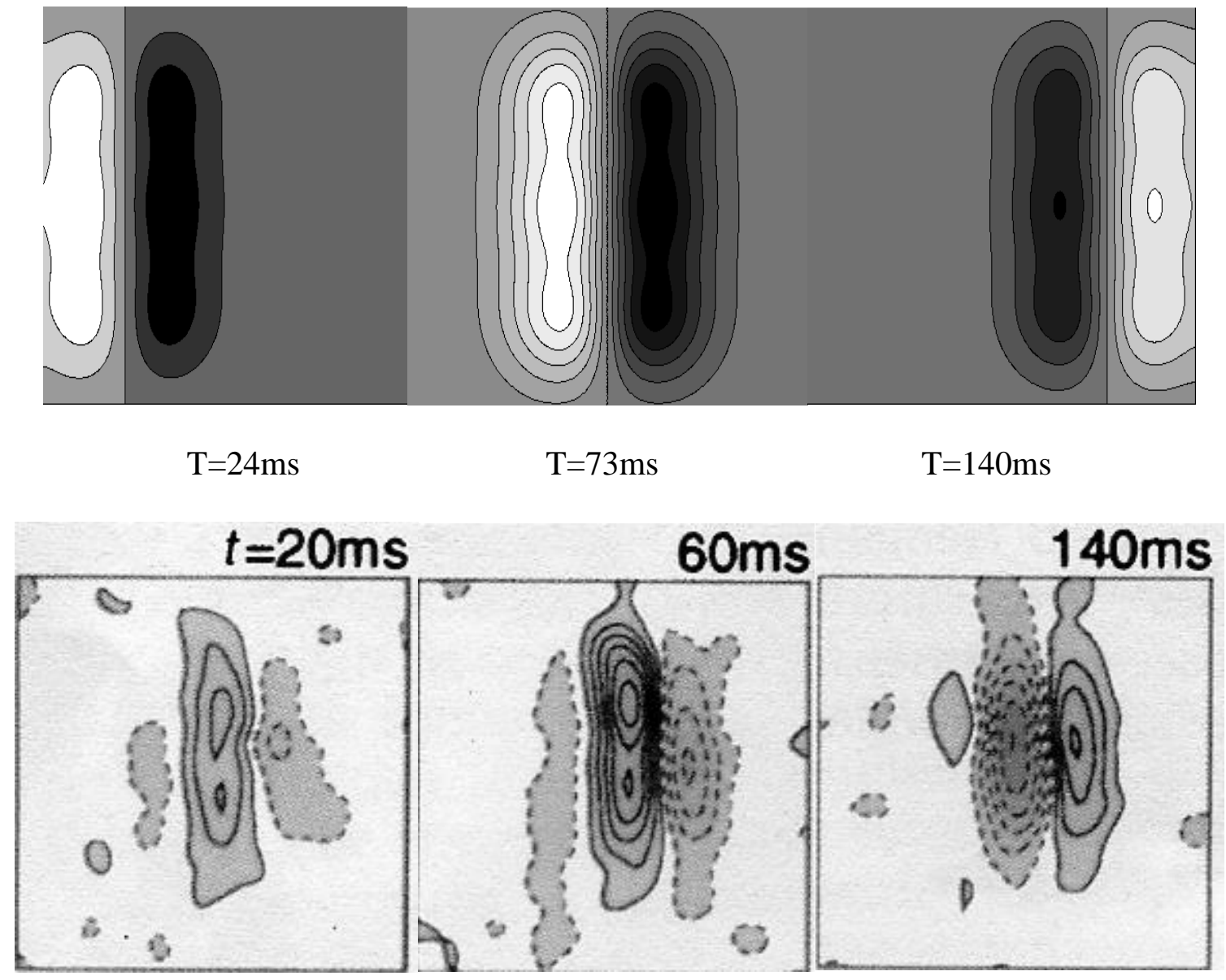

Fig (19): (top) Spatio-temporal response of a non-isotopic velocity adapted neuron shown in figure (16-bottom) for $x_{d}=10, y_{d}=10, n=10, R_{z} C_{z}=1.9, \frac{G_{z}}{C_{z}}=0.001, R_{z} C_{z} z^{2}=400$ and $\mu=130$ (bottom) Examples of spatiotemporal receptive field profile of a non-separable (velocity adapted) non-isotropic neuron as reported by DeAngelis et al. (1995). 

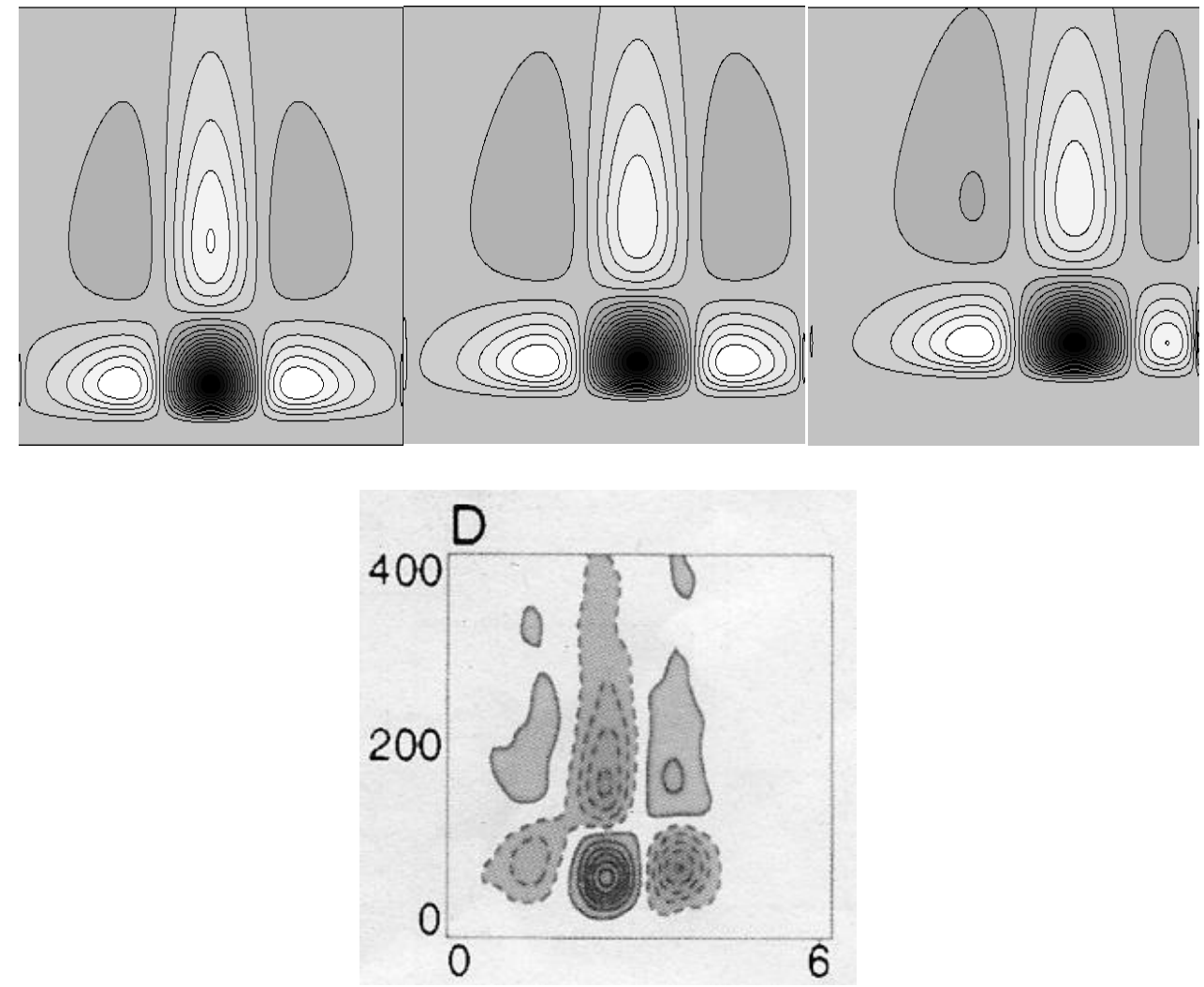

Fig (20): (top) Spatio-temporal responses $h_{x x t}$ of three neurons with a configuration similar to those shown as the red neurons in figure (16-bottom) with separable filters, (bottom) An example of the 1+1D spatiotemporal receptive profile of a neuron reminiscent of a first-order derivative in time and a second-order derivative in space as reported by DeAngelis et al. (1995). 

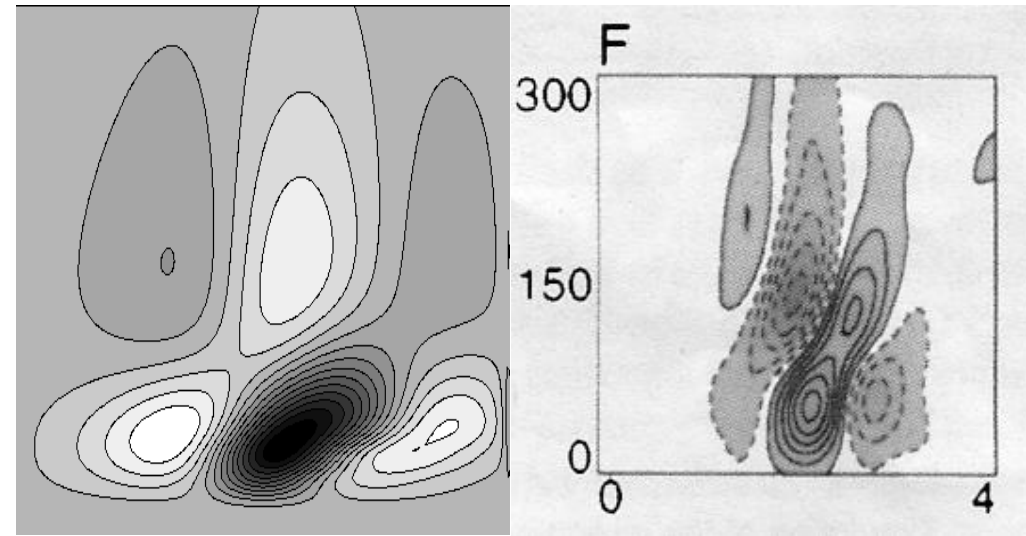

Fig (21): (left) Velocity-adapted non-separable 1+1D spatio-temporal response produced by summing the three separable spatio-temporal responses shown in figure (20-top) (right) the spatiotemporal receptive profile in tilted space-time as reported in (DeAngelis et al. 1995). 

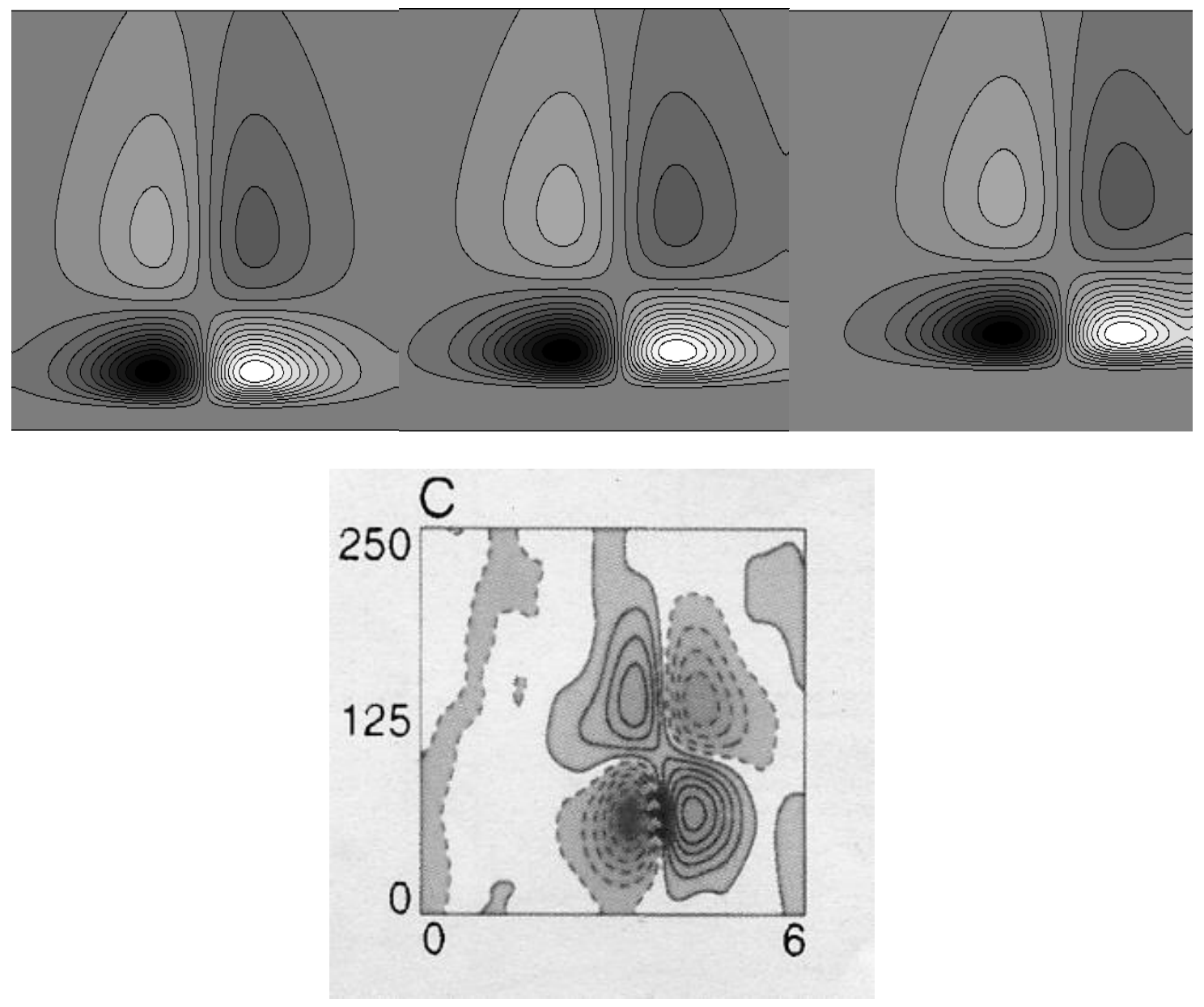

Fig (22): (top) Spatio-temporal responses $h_{x t}$ of three neurons with a configuration similar to that shown as the red neurons in figure (16-bottom) with separable filters, (bottom) An example of spatiotemporal receptive field profile of a simple cell in the striate cortex reminiscent of a first order derivative in space and a first derivative in time as reported in (DeAngelis et al. 1995) 

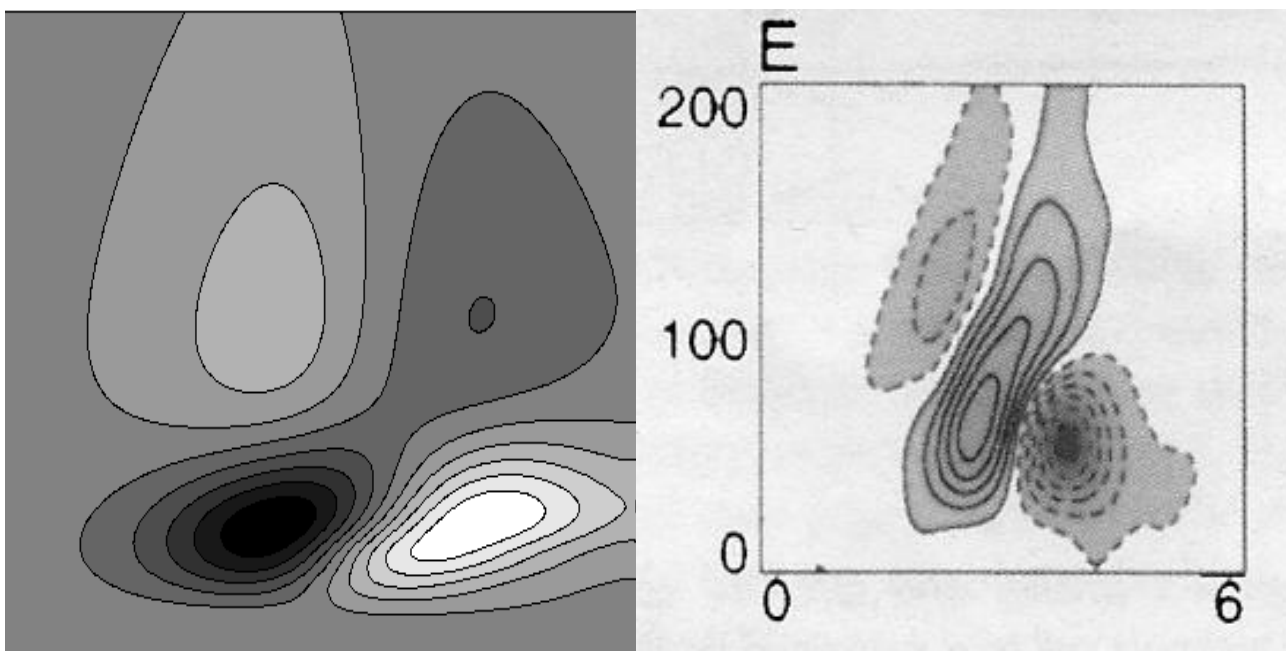

Fig (23): (left) Velocity-adapted non-separable 1+1D spatio-temporal response produced by summing the three separable spatio-temporal responses shown in figure (22-top), (right) An example of biologically recorded spatiotemporal receptive field profile of a simple cell as reported in (DeAngelis et al. 1995). 